\title{
TORQUE-LIMITED GROWTH OF MASSIVE BLACK HOLES IN GALAXIES ACROSS COSMIC TIME
}

\author{
Daniel Anglés-Alcázar ${ }^{1,2}$, Feryal ÖZel ${ }^{3}$, Romeel Davé ${ }^{4,5,6}$, Neal Katz $^{7}$, \\ Juna A. KOllmeier ${ }^{8}$, AND BenJamin D. OPPENHEIMER ${ }^{9}$ \\ ${ }^{1}$ Department of Physics, University of Arizona, Tucson, AZ 85721, USA; anglesd@email.arizona.edu \\ ${ }^{2}$ Center for Interdisciplinary Exploration and Research in Astrophysics (CIERA) and Department of Physics and Astronomy, \\ Northwestern University, Evanston, IL 60208, USA \\ ${ }^{3}$ Astronomy Department, University of Arizona, Tucson, AZ 85721, USA \\ ${ }^{4}$ University of the Western Cape, Bellville, Cape Town 7535, South Africa \\ ${ }^{5}$ South African Astronomical Observatories, Observatory, Cape Town 7925, South Africa \\ ${ }^{6}$ African Institute for Mathematical Sciences, Muizenberg, Cape Town 7945, South Africa \\ ${ }^{7}$ Astronomy Department, University of Massachusetts, Amherst, MA 01003, USA \\ ${ }^{8}$ Observatories of the Carnegie Institute of Washington, Pasadena, CA 91101, USA \\ ${ }^{9}$ CASA, Department of Astrophysical and Planetary Sciences, University of Colorado, Boulder, CO 80309, USA \\ Received 2013 September 20; accepted 2014 December 15; published 2015 February 20
}

\begin{abstract}
We combine cosmological hydrodynamic simulations with analytic models to evaluate the role of galaxy-scale gravitational torques on the evolution of massive black holes at the centers of star-forming galaxies. We confirm and extend our earlier results to show that torque-limited growth yields black holes and host galaxies evolving on average along the $M_{\mathrm{BH}}-M_{\text {bulge }}$ relation from early times down to $z=0$ and that convergence onto the scaling relation occurs independent of the initial conditions and with no need for mass averaging through mergers or additional self-regulation processes. Smooth accretion dominates the long-term evolution, with black hole mergers with mass ratios $\gtrsim 1: 5$ representing typically a small fraction of the total growth. Winds from the accretion disk are required to eject significant mass to suppress black hole growth, but there is no need for coupling this wind to galactic-scale gas to regulate black holes in a nonlinear feedback loop. Torque-limited growth yields a close-to-linear $\left\langle\dot{M}_{\mathrm{BH}}\right\rangle \propto$ star formation rate (SFR) relation for the black hole accretion rate averaged over galaxy evolution timescales. However, the SFR-AGN connection has significant scatter owing to strong variability of black hole accretion at all resolved timescales. Eddington ratios can be described by a broad lognormal distribution with median value evolving roughly as $\lambda_{\mathrm{MS}} \propto(1+z)^{1.9}$, suggesting a main sequence for black hole growth similar to the cosmic evolution of specific SFRs. Our results offer an attractive scenario consistent with available observations in which cosmological gas infall and transport of angular momentum in the galaxy by gravitational instabilities regulate the long-term co-evolution of black holes and star-forming galaxies.
\end{abstract}

Key words: black hole physics - galaxies: active - galaxies: evolution - quasars: general

\section{INTRODUCTION}

A wide range of observations imply a close connection between central massive black holes and their host galaxies, including the similarity between the cosmic star formation history and the evolution of global black hole accretion (Madau et al. 1996; Boyle \& Terlevich 1998; Hopkins \& Beacom 2006; Silverman et al. 2008; Aird et al. 2010; Rodighiero et al. 2010), the higher incidence of active galactic nuclei (AGNs) in highermass galaxies and strongly star-forming systems (Kauffmann et al. 2003; Silverman et al. 2009; Rafferty et al. 2011; Santini et al. 2012; Juneau et al. 2013; Rosario et al. 2013; Trump et al. 2013), as well as a number of correlations between the mass of the central black hole and properties of the host galaxy such as the stellar mass of the central bulge $\left(M_{\mathrm{BH}}-M_{\text {bulge }}\right.$ relation; Magorrian et al. 1998; Häring \& Rix 2004; Scott et al. 2013), and its velocity dispersion (Ferrarese \& Merritt 2000; Tremaine et al. 2002; Gültekin et al. 2009; Graham et al. 2011; McConnell et al. 2011). This circumstantial evidence has led many to conclude that massive black holes play a key role in galaxy evolution (Somerville et al. 2008; Cattaneo et al. 2009) and yet, unravelling the physical mechanisms driving this connection remains one of the major unsolved problems in modern astrophysics.

The growth of massive black holes at the centers of galaxies involves a remarkable variety of physical processes operating at scales ranging from the size of the entire galaxy down to the black hole event horizon (see Alexander \& Hickox 2012, for a review). In a broad view, the procedure for growing black holes involves (1) feeding the black hole from the accretion disk; (2) the regulation of growth owing to feedback processes (winds and thermal pressure); and (3) the supply of gas from the galaxy onto the accretion disk.

An accretion flow forms in the region where the potential of the black hole dominates that of the galaxy and angular momentum is transported outward by turbulent MHD processes (Shakura \& Sunyaev 1973; Balbus \& Hawley 1998). The rate at which gas inflows through the sphere of influence of the black hole is believed to determine the overall geometry and radiative properties of the accretion flow (see, e.g., Abramowicz \& Fragile 2013 for a recent review). In different accretion rate regimes, analytic arguments and numerical simulations show that a significant fraction of the inflowing mass is likely to be lost to winds and outflows (see, e.g., Blandford \& Payne 1982; Narayan \& Yi 1995a; Proga et al. 2000; Narayanan et al. 2006; Ohsuga \& Mineshige 2011; Sadowski et al. 2013). Moreover, observations show that winds and outflows are frequent in AGNs (Reynolds 1997; Veilleux et al. 2005; Fabian 2012) and may carry significant amounts of mass away (e.g., King et al. 2013). Indeed, powerful galactic-scale molecular gas outflows thought to be driven by nuclear activity are observed both in the local and the high-redshift universe, and the total mass loss rate 
may exceed the star formation rate (SFR) of the entire galaxy (Feruglio et al. 2010; Rupke \& Veilleux 2011; Sturm et al. 2011; Maiolino et al. 2012). Thus, winds and outflows powered by black hole accretion could represent a significant mass loss relative to the inflowing gas from larger scales.

The impact of this outflowing gas on the scale of the galaxy has received considerable attention recently, as a way to further regulate black hole growth by actively affecting the rate at which gas inflows feed the accretion disk from galactic scales. If the interaction between inflows and the outflowing mass and radiation is strong enough, this "AGN feedback" may be the primary modulator of long term black hole growth (e.g., Fabian 2012). In this scenario, black hole growth becomes selfregulated, the feedback coupling efficiency represents the key physical process, and the mechanism responsible for driving gas inflows from galactic scales down to the accretion flow becomes sub-dominant. This paradigm has been extensively explored both in analytic models (e.g., Silk \& Rees 1998; King 2003; Murray et al. 2005) and numerical simulations (Di Matteo et al. 2005, 2008; Hopkins et al. 2006; Robertson et al. 2006b; Hopkins et al. 2007; Booth \& Schaye 2009; Dubois et al. 2012) with significant success in explaining a variety of observations including the black hole-galaxy scaling relations.

AGN feedback may also have a strong impact on the host galaxy and possibly be responsible for the observed exponential cutoff at the high mass end of the stellar mass function (Baldry et al. 2008) and the observed dichotomy between blue star-forming galaxies and red quiescent galaxies (Schawinski et al. 2007). Indeed, AGN feedback is often invoked in semianalytic models (e.g., Bower et al. 2006; Croton et al. 2006; Somerville et al. 2008) and hydrodynamic simulations (e.g., Springel et al. 2005; Gabor et al. 2011; Teyssier et al. 2011; Dubois et al. 2013; Puchwein \& Springel 2013) as an additional energy source to suppress cooling flows and star formation in early-type galaxies. There remain, however, significant concerns relative to the overall efficiency of feedback required by self-regulated models (e.g., Silk \& Nusser 2010), the interplay between AGN feedback and stellar feedback (e.g., Cen 2012), and the intrinsic degeneracy often suffered by coupled accretion-feedback models (Newton \& Kay 2013; Wurster \& Thacker 2013).

In comparison, the physical processes responsible for feeding the black hole accretion disk in the first place have received comparably little attention. Most numerical investigations have relied on the Bondi-Hoyle-Littleton accretion prescription (Hoyle \& Lyttleton 1939; Bondi \& Hoyle 1944; Bondi 1952) to capture gas from the inner galaxy and feed the black hole accretion disk (e.g., Di Matteo et al. 2005; Booth \& Schaye 2009). However, this prescription does not account for the rate at which angular momentum can be lost by the infalling gas, which could easily be the limiting factor for fuelling AGNs (Jogee 2006). Hence, the physical mechanisms driving the required continuous supply of gas from galactic scales down to subparsec scales may play a more crucial role than commonly considered (Escala 2006, 2007).

Hydrodynamic simulations of gas-rich galaxy mergers have shown that large-scale tidal torques induced by the interaction, or even gravitational instabilities in self-gravitating disks, can lead to angular momentum transport and the rapid inflow of gas to the central $\sim 100 \mathrm{pc}$ of galaxies (Hernquist 1989; Shlosman et al. 1989; Barnes \& Hernquist 1992; Escala 2007; Hopkins \& Quataert 2010). Another alternative for AGN fuelling is direct clump-clump interactions in turbulent gas-rich disks at high redshift (Bournaud et al. 2011; Gabor \& Bournaud 2013). However, subsequent gravitational instabilities become less efficient at scales comparable to the black hole radius of influence, $\sim 10 \mathrm{pc}$, requiring additional mechanisms to transport gas down to smaller scales (Jogee 2006). Furthermore, the gas is still self-gravitating at these scales and, therefore, likely to participate in star formation (Thompson et al. 2005). Using multiple nested simulations of progressively higher resolution, Hopkins \& Quataert (2010, 2011) showed that non-axisymmetric perturbations to the stellar potential may induce strong orbit crossing, driving gas into shocks that dissipate energy and angular momentum, and providing significant gas inflows down to $\sim 0.01 \mathrm{pc}$ scales.

In this paper, we evaluate the role of black hole feeding limited by galaxy-scale gravitational torques on the evolution of massive black holes at the centers of star-forming galaxies over cosmic time, minimizing the assumptions made on the effects of AGN feedback on galactic scales. In our previous work (Anglés-Alcázar et al. 2013), we combined cosmological zoom simulations of galaxy formation down to $z=2$ together with analytic parametrizations of black hole growth to show that a model in which black hole growth is limited by galaxyscale torques (Hopkins \& Quataert 2011) does not require selfregulation of black hole growth. Specifically, torque-limited growth yields black holes and galaxies evolving on average along the observed scaling relations from early times down to $z \sim 2$, providing a plausible scenario to explain their connection that does not crucially invoke AGN feedback. Winds from the accretion disk are still required in this scenario to drive significant mass loss from the accretion disk (roughly 95\%), thereby strongly suppressing black hole growth, but there is no need to strongly couple these winds to galaxy-scale gas to regulate black hole growth in a nonlinear feedback loop. This removes the need for self-regulation via spherical feedback as commonly assumed in Bondi accretion-based models $;{ }^{10}$ instead, the wind can propagate biconically from the accretion disk and be weakly coupled to the inflow at a sub-resolution level, which is perhaps more physically plausible for black hole growth within disk galaxies.

Motivated by the attractive features of the torque-limited growth model, we extend the analysis in Anglés-Alcázar et al. (2013) to examine black hole growth in a larger population of galaxies down to $z=0$ by employing full cosmological hydrodynamic simulations. We describe the simulations and the overall methodology in Section 2 and report our main results in Section 3. We present resolution convergence tests to show the robustness of our methodology in Section 4, and we conclude in Section 5 by discussing implications in the context of current theoretical models and observations.

\section{METHODOLOGY}

We apply and extend the methodology described in AnglésAlcázar et al. (2013) to follow the growth of massive black holes over cosmic time. We begin by identifying a population of galaxies at $z=0$ from a full cosmological hydrodynamic simulation and characterize their evolution back in time. Then, we infer how black holes grow at the centers of galaxies in post-processing, by evaluating accretion rates based on the gravitational torque model of Hopkins \& Quataert (2011), and accounting for the mass growth through black hole mergers.

\footnotetext{
10 See Dubois et al. (2012) for a non-isotropic kinetic-mode feedback model capable of self-regulating black hole growth in the context of Bondi accretion.
} 


\subsection{Simulations}

We use an extended version of the $N$-body + smoothed particle hydrodynamics cosmological galaxy formation code GADGET-2 (Springel 2005) to simulate the evolution of a $\left[32 h^{-1} \mathrm{Mpc}\right]^{3}$ comoving volume down to $z=0$. Our primary simulation utilizes $2 \times 512^{3}$ gas + dark matter particles with masses $m_{\text {gas }} \approx$ $4.5 \times 10^{6} M_{\odot}$ and $m_{\mathrm{DM}} \approx 2.3 \times 10^{7} M_{\odot}$, respectively, and a fixed comoving softening length $\epsilon \approx 1.25 h^{-1} \mathrm{kpc}$. Throughout this paper we assume a $\Lambda \mathrm{CDM}$ concordance cosmology with parameters $\Omega_{\Lambda}=0.72, \Omega_{\mathrm{M}}=0.28, \Omega_{\mathrm{b}}=0.046, h=0.7$, $\sigma_{8}=0.82$, and $n=0.96$, consistent with the latest nineyear Wilkinson Microwave Anisotropy Probe data (Hinshaw et al. 2013).

Our main simulation was first described in Davé et al. (2013). We include radiative cooling from primordial gas (Katz et al. 1996), metal-line cooling (Sutherland \& Dopita 1993), and photoionization heating from an optically thin UV background (Haardt \& Madau 2001) starting at $z=9$. Star formation is modeled probabilistically through a multi-phase sub-grid prescription (Springel \& Hernquist 2003) where gas particles that are sufficiently dense to become Jeans unstable can spawn a star particle with a probability based on a Schmidt (1959) law. The resulting SFRs are tuned to be in accord with the observed Kennicutt (1998) relation. We include metal enrichment from Type Ia and Type II supernovae (SNe) and asymptotic giant branch (AGB) stars, energy feedback from Type Ia and Type II SNe, and mass-loss from AGB stars as described in Oppenheimer \& Davé (2006, 2008). We assume a Chabrier (2003) initial mass function throughout.

Galactic outflows are modeled by imparting kinetic energy to gas particles with a probability given by the mass loading factor $(\eta)$ times the star formation probability. Outflow velocities scale with galactic velocity dispersion $(\sigma)$ and the mass loading factor scales as $\eta \propto 1 / \sigma$ (as in the momentum-driven case) and $\eta \propto 1 / \sigma^{2}$ (as in the energy-driven case) for galaxies above and below $\sigma=75 \mathrm{~km} \mathrm{~s}^{-1}$, respectively (Davé et al. 2013). This is motivated by recent analytic models (Murray et al. 2010) as well as galaxy-scale hydrodynamic simulations with explicit stellar feedback models (Hopkins et al. 2012). Our primary simulation also incorporates a heuristic prescription to quench star formation that is tuned to reproduce the observed exponential cutoff in the high-mass end of the stellar mass function at $z=0$ (Davé et al. 2013). This ad hoc quenching prescription has no major effect on our results, as we show in Section 4.

Note that we do not attempt to explicitly model AGN feedback in our simulations. Instead, we focus on the role of feeding black holes by galaxy-scale gravitational torques and use the observed connections between central black holes and host galaxies to put constraints on the overall impact of AGN feedback.

\subsection{Host Galaxies}

We produce 135 redshift snapshots from $z=30$ down to $z=0$. Following Anglés-Alcázar et al. (2013), we identify individual galaxies in each snapshot as bound collections of star-forming gas and star particles by means of the Spline Kernel Interpolative Denmax algorithm (SKID ${ }^{11}$ ). Each SKID-identified galaxy is associated with a dark matter halo by using a spherical overdensity algorithm, where the virial radius is defined to enclose a mean density given by Kitayama \& Suto (1996).

\footnotetext{
11 http://www-hpcc.astro.washington.edu/tools/skid.html
}

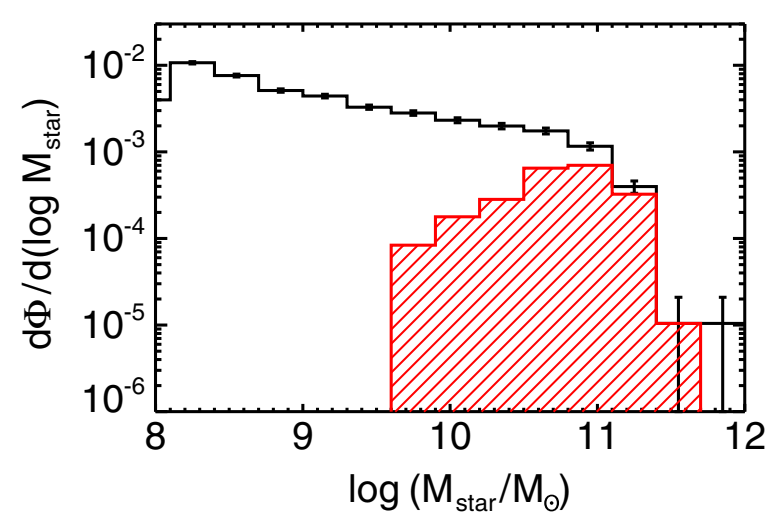

Figure 1. Galaxy stellar mass function at $z=0$ (black). The red hatched area corresponds to the primary galaxy sample used in this work.

Overlapping halos are merged together so that every final halo contains one central galaxy (the most massive galaxy) and a number of satellite galaxies by construction.

We follow the evolution of central galaxies back in time beginning at $z=0$ by identifying their most massive progenitor at each previous snapshot. The main progenitor at time $t$ is defined as the galaxy with the highest fraction of the total stellar mass of a given galaxy at time $t+\Delta t$. Only a sub-sample of all central galaxies identified at $z=0$ is used in our primary analysis. Unless otherwise noted, we require galaxies to contain at least 200 gas and 200 star particles at all times and to be identified in the cosmological simulation as early as $z \geqslant 4$. This selection criteria allows us to characterize the morphological properties of galaxies and to evaluate the evolution of their central black holes for a cosmologically significant period of time. Nonetheless, in Sections 3.5 and 4 we will enlarge our Galaxy sample to expand the dynamic range for a few particular redshifts.

Figure 1 shows the stellar mass function for all galaxies in our $\left[32 h^{-1} \mathrm{Mpc}\right]^{3}$ simulated volume, where the red hatched area indicates the primary sub-sample of 213 galaxies selected for this work. As expected, the requirement for galaxies to be resolved in the simulation at $z \geqslant 4$ results in a sub-sample containing mainly massive galaxies. Note that the requirement for a minimum number of gas particles eliminates eleven massive galaxies with extremely low gas fractions at low redshift.

\subsection{Black Hole Seeds}

Several alternative scenarios have been proposed for the formation of primordial seeds that eventually become the massive black holes populating the centers of galaxies (for a review, see Volonteri 2010). Popular models include the formation of light seeds $\left(\sim 10^{2} M_{\odot}\right)$ as remnants of population III stars (e.g., Madau \& Rees 2001) and the formation of massive black holes $\left(\sim 10^{5} M_{\odot}\right)$ by direct collapse in pre-galactic halos (e.g., Begelman et al. 2006; Choi et al. 2013). Despite much theoretical work, major uncertainties remain on the initial mass of black hole seeds, their birth places and number densities, and their formation redshift.

A common feature of current theoretical models is the requirement of large amounts of pristine gas only available at very high redshifts $(z \gtrsim 15)$. Since our simulations do not resolve galaxies until $z \approx 8$ even for the most massive systems, we have to populate galaxies with black holes that have been presumably evolving within their hosts for at least a few hundred 
million years. The simplest approach is, therefore, to assume that there is only one central black hole by the time we first resolve each galaxy and that its mass scales with the stellar mass of the host galaxy in a way similar to the observed $z=0 M_{\mathrm{BH}}-M_{\text {bulge }}$ relation (dual AGNs are usually associated with merger systems, at least at low redshift; see, e.g., Comerford et al. 2009).

We assign a seed black hole to every galaxy by assuming consistency with the $M_{\mathrm{BH}}-M_{\text {bulge }}$ relation of Häring \& Rix (2004) evaluated for the stellar mass within the effective radius of the host galaxy, regardless of the redshift when it is first resolved in the simulation. We also perform tests by assigning initial black hole masses either a factor of 10 above or below the scaling relation, or drawn from a log-normal distribution with a mean and a dispersion similar to that of the observed local $M_{\mathrm{BH}}-M_{\text {bulge }}$ relation. We will justify the assumption of correlated initial conditions in the torque-limited growth model, since we will show that it yields black holes that evolve toward the observed scaling relations independent of their initial conditions (Anglés-Alcázar et al. 2013).

\subsection{Accretion Rates}

Once a seed black hole has been assigned to a given galaxy, accretion rates are calculated based on the gravitational torque rate introduced by Hopkins \& Quataert (2011), $\dot{M}_{\text {Torque }}$, assuming that only a fraction $\epsilon_{\mathrm{m}}$ of the inflowing gas at subparsec scales is actually accreted by the black hole, with the rest lost to winds and outflows (Anglés-Alcázar et al. 2013):

$$
\frac{d M_{\mathrm{BH}}}{d t}=\epsilon_{\mathrm{m}} \dot{M}_{\text {Torque }}(t) .
$$

The gravitational torque model predicts gas inflow rates from galactic scales to sub-parsec scales as a function of galaxy properties evaluated within a radial aperture, $R_{0}$, that must be resolved in the cosmological simulation (Hopkins \& Quataert 2011):

$$
\begin{aligned}
\dot{M}_{\text {Torque }} \approx & \alpha_{\mathrm{T}} f_{\mathrm{d}}^{5 / 2} \times\left(\frac{M_{\mathrm{BH}}}{10^{8} M_{\odot}}\right)^{1 / 6}\left(\frac{M_{\mathrm{d}}\left(R_{0}\right)}{10^{9} M_{\odot}}\right) \\
& \times\left(\frac{R_{0}}{100 \mathrm{pc}}\right)^{-3 / 2}\left(1+\frac{f_{0}}{f_{\text {gas }}}\right)^{-1} M_{\odot} \mathrm{yr}^{-1},
\end{aligned}
$$

where

$$
\begin{gathered}
f_{\mathrm{d}} \equiv M_{\mathrm{d}}\left(R_{0}\right) /\left(M_{\mathrm{gas}}\left(R_{0}\right)+M_{\text {star }}\left(R_{0}\right)\right), \\
f_{\text {gas }} \equiv M_{\text {gas }}\left(R_{0}\right) / M_{\mathrm{d}}\left(R_{0}\right), \\
f_{0} \approx 0.31 f_{\mathrm{d}}^{2}\left(M_{\mathrm{d}}\left(R_{0}\right) / 10^{9} M_{\odot}\right)^{-1 / 3},
\end{gathered}
$$

and $M_{\mathrm{d}}\left(R_{0}\right)$ is the total (gas+stars) disk mass within $R_{0}$, $M_{\text {gas }}\left(R_{0}\right)$ and $M_{\text {star }}\left(R_{0}\right)$ represent the total gas and stellar masses within $R_{0}$, and $\alpha_{\mathrm{T}} \approx 5$ is a normalization factor that parametrizes the dependence of inflow rates on star formation at scales not resolved (Hopkins \& Quataert 2011; Anglés-Alcázar et al. 2013).

To estimate the disk mass within $R_{0}$ for the gas and stellar components, $M_{\mathrm{d}}$, we perform a simple bulge-disk kinematic decomposition using the full three-dimensional information available in the simulations. Recent morphological studies of simulated galaxies have identified two distinct dynamical components in the distribution of the rotational support of their baryonic content, clearly associated with the disk and bulge morphological components (e.g., Abadi et al. 2003; Governato et al. 2009; Hopkins et al. 2009; Scannapieco et al. 2009; Christensen et al. 2014). Motivated by these studies, we calculate the azimuthal velocity $v_{\phi}$ of each particle with respect to the direction of the total angular momentum within $R_{0}$, and estimate the mass in a spheroidal component, $M_{\text {bulge }}\left(R_{0}\right)$, as double the mass of particles moving with $v_{\phi}<0$. The disk mass is, then, $M_{\mathrm{d}}\left(R_{0}\right) \approx M_{\text {tot }}\left(R_{0}\right)-M_{\text {bulge }}\left(R_{0}\right)$, where $M_{\text {tot }}\left(R_{0}\right)=M_{\text {gas }}\left(R_{0}\right)+M_{\text {star }}\left(R_{0}\right)$ is the total mass within $R_{0}$. Note that this kinematic decomposition is formally equivalent to that performed in Abadi et al. (2003) based on the distribution of the orbital circularity parameter. The basic assumption is that the spheroid has little net rotation, with as many gas/star particles in co- as in counterrotating orbits. This will certainly overestimate $f_{\mathrm{d}}$ in the case of rotating bulges but it is a reasonable approximation for the purpose of evaluating Equation (2). While several different bulge-disk decomposition procedures are possible, our main results are qualitatively independent of the exact definition of the bulge and disk components. Any quantitative differences could be in principle absorbed into the normalization factor $\epsilon_{\mathrm{m}}$, and, as we show in the Appendix, our bulge-disk decomposition procedure shows better resolution convergence relative to other methods.

In our previous work, we found that a constant radial aperture $R_{0}=1 \mathrm{kpc}$ to be appropriate for all galaxies at all times, since kiloparsec scales were well resolved in our cosmological zoom simulations (Anglés-Alcázar et al. 2013, 2014). This fixed radial aperture is likely not appropriate here given the significantly larger range of galaxy masses and evolution times. Instead, we adopt a variable, time-dependent $R_{0}$ defined to be the smallest radial aperture containing at least 200 gas particles and 200 star particles. With this definition, we ensure that physical quantities such as gas fraction and disk fraction entering into the calculation of gravitational torque rates can be appropriately characterized for all galaxies at all times. In Section 4, we evaluate the effects of using different radial apertures on the inferred black hole accretion rates, and show that it has only a modest impact.

We calculate the growth of black holes through direct smooth gas accretion by numerical integration of Equation (1) for the initial black hole mass defined for each galaxy (Section 2.3). The integration time step is constrained by the number of redshift snapshots available, ranging in frequency from $\sim 10$ to $300 \mathrm{Myr}$ in the redshift range $z \sim 6 \rightarrow 0$, i.e., $\lesssim 2 \%$ of the Hubble time at any given redshift. Inferred black hole accretion rates represent, therefore, average values for the corresponding time steps. The gravitational torque rate, $\dot{M}_{\text {Torque }}(t)$, is calculated based on the physical properties of each galaxy at a given time (Equation (2)) and is evaluated with the appropriate black hole mass at each time step, as given by Equation (1).

Note that by evaluating Equation (1) in post-processing we are neglecting the gravitational influence of the central black hole at the scales resolved in the simulation. This is unlikely to affect our results since we are considering the transport of angular momentum at scales well beyond the black hole radius of influence. In addition, we assume that outflows powered by black hole accretion are weakly coupled to the gas inflows and do not alter significantly the evaluation of gravitational torque rates (Anglés-Alcázar et al. 2013). Equation (1) implies that a total mass $M_{\text {out }} \approx\left(1 / \epsilon_{\mathrm{m}}-1\right) \times M_{\mathrm{BH}}$ will be ejected from the accretion disk during the full evolution of the central black hole, though not necessarily leaving the host galaxy. This represents 
$\sim 2 \%$ of the final stellar mass of the host galaxy, simply assuming $M_{\mathrm{BH}} \sim M_{\mathrm{star}} / 1000$ and $\epsilon_{\mathrm{m}}=5 \%$ (see Section 3.2). In contrast, for a mass loading factor $\eta \sim 2$, star formation driven winds in our simulation will have ejected at least 100 times more gas than the direct mass loss owing to accretion-driven winds (assuming no significant entrainment of cold interstellar medium (ISM) gas). It is, thus, reasonable to treat the overall mass loss $\epsilon_{\mathrm{m}}$ as a zeroth order effect on black hole growth and neglect any higher order effects for the purpose of evaluating $\dot{M}_{\text {Torque }}$.

\subsection{Black Hole Mergers}

In addition to black hole growth by torque-limited accretion, we evaluate the mass growth rate from black hole mergers. If a major merger is identified for a given central galaxy, we assume that the merging galaxy contains a black hole consistent with the $M_{\mathrm{BH}}-M_{\text {bulge }}$ relation of Häring \& Rix (2004) and we add its corresponding mass to the total mass of the final black hole in the remnant galaxy. We also incorporated an alternate prescription as with black hole seeding, where the mass of the merging black hole is chosen randomly from a log-normal distribution corresponding to the $M_{\mathrm{BH}}-M_{\text {bulge }}$ relation for its host galaxy and added that mass to the central black hole accordingly. Any time delay between the merger of the host galaxies and the final merger of their central black holes (e.g., Dubois et al. 2010) is neglected for the sake of simplicity. This is unlikely to affect our results given the weak dependence of gravitational torque rates on black hole mass.

We limit ourselves to major galaxy mergers where the mass ratios of interacting galaxies are above 1:5. If $M_{*}(t+\Delta t)$ is the total stellar mass of a central galaxy at time $t+\Delta t$ (where $\Delta t$ represents the time interval between simulations outputs) and $M_{*}^{1 \text { st }}(t)$ and $M_{*}^{2 \text { nd }}(t)$ are the stellar masses of its first and second most massive progenitors at time $t$, major galaxy mergers $(>1: 5)$ are identified by the following criteria:

$$
\begin{aligned}
& \text { 1. } M_{*}^{2 \text { nd }}(t) \geqslant 1 / 5 \times M_{*}^{1 \mathrm{st}}(t) \\
& \text { 2. } M_{*}(t+\Delta t)>(1+1 / 5) M_{*}^{1 \mathrm{st}}(t) \\
& \text { 3. } M_{*}(t+\Delta t)>0.8\left(M_{*}^{1 \mathrm{st}}(t)+M_{*}^{2 \mathrm{nd}}(t)\right) \\
& \text { 4. } \min \left\{\Delta M_{*}\right\}_{t \rightarrow t+600 \mathrm{Myr}}>-0.5 M_{*}^{2 \mathrm{nd}}(t) .
\end{aligned}
$$

The identification of galaxy mergers in cosmological simulations is not a trivial task, where the simple working definition of "galaxy" can, for example, result in the wrong identification of close galaxy encounters as a merging system (e.g., Gabor et al. 2011). We determined and tested the above criteria experimentally by comparing the identified merger events against the evolution of the stellar mass of central galaxies relative to that of their most massive progenitors to ensure that close encounters are not treated as mergers.

The first and second conditions reflect our definition of major mergers $(>1: 5)$ and the requirement that the central galaxy has indeed grown by at least one fifth relative to its stellar mass in the previous time step. Note that the mass increase $\Delta M_{*}=M_{*}(t+\Delta t)-M_{*}(t)$, where $M_{*}(t) \equiv M_{*}^{1 \text { st }}(t)$, contains contributions from both major and minor mergers as well as star formation within the galaxy. The third condition, requiring that the merger remnant contains at least $80 \%$ of the mass of its two most massive progenitors, is apparently less restrictive than the second condition; however, it accounts for situations in which $M_{*}^{2 \text { nd }}(t)>M_{*}^{1 \text { st }}(t)$ that may occur if only a small fraction of $M_{*}^{2 \text { nd }}(t)$ ends up in the merger remnant. Finally, the fourth condition attempts to correct for wrong identifications during close galaxy encounters by requiring that any decrease in stellar mass during the $\sim 600 \mathrm{Myr}$ after the merger cannot be higher than half the mass of the second most massive progenitor.

At all times, gravitational torque rates (Equation (2)) are evaluated according to the current mass of the black hole including contributions from mergers. Note that we neglect the possibility of black holes leaving the center of their host galaxies owing to gravitational recoils (e.g., Blecha \& Loeb 2008).

\section{RESULTS}

\subsection{Black Hole Mergers versus Smooth Accretion}

Figure 2 illustrates the identification of galaxy mergers, based on the criteria described in Section 2.5, by showing the evolution of the total stellar mass of nine representative galaxies in the mass range $M_{\text {star }}=10^{10}-2 \times 10^{11} M_{\odot}$ at $z=0$. Major merger events are indicated by red vertical lines and correspond to abrupt changes in the stellar mass of the galaxies. Note that the time interval between data snapshots varies with redshift, implying that the mass increase per unit time required for merger identification is redshift dependent. This could result in an increasing number of merger identifications at lower redshifts owing, for example, to contributions from smooth accretion and minor mergers occurring in a single (longer) time step. However, this is compensated by the shorter time steps at the epoch near the peak of cosmic star formation activity $(z \sim 2)$.

Galaxy misidentifications by SKID represent a more challenging issue (e.g., Gabor et al. 2011). Interacting galaxies are sometimes identified as one single galaxy at the closest approach during the first orbital passage, with a consequent increase in the stellar mass of the newly identified central galaxy. When the distance between the interacting galaxies increases again, two separate systems are identified and the mass of the central galaxy decreases correspondingly. The fourth condition for merger identification in Section 2.5 attempts to correct for this effect. We present examples of this for several galaxies in Figure 2.

Overall, our simple method provides a robust identification of galaxy mergers and allows us to estimate the contribution of black hole mergers to total black hole growth. For each galaxy in Figure 2, the total mass of the central black hole as a function of redshift is shown as the blue line (upscaled by a factor of 1000), while the gray lines correspond to black hole growth from torque-limited accretion only. Here we adopt a mass retention rate $\epsilon_{\mathrm{m}}=0.05$, which has been shown to reproduce the normalization of the $M_{\mathrm{BH}}-M_{\text {bulge }}$ relation at $z \geqslant 2$ (AnglésAlcázar et al. 2013), and assume that the merging galaxy contains a black hole consistent with the local $M_{\mathrm{BH}}-M_{\text {bulge }}$ relation of Häring \& Rix (2004). We relax this assumption in Section 3.2 by considering a 0.5 dex scatter in black hole mass.

The most massive black holes are expected to undergo more frequent mergers as their host galaxies also represent the high-mass end of the galaxy mass distribution and live in higher density environments. Indeed, the left panel of Figure 3 shows that our sub-sample of lower mass galaxies clearly dominates the population of galaxies that undergo only one or no major mergers during their entire evolution down to $z=0$. Correspondingly, galaxies in the higher-mass sub-sample tend to undergo two or more major mergers down to $z=0$.

The contribution from each black hole merger represents a significant fraction of the total black hole mass at the time of the merger event, typically $\gtrsim 20 \%$ given that we define major galaxy mergers to be mass ratios above 1:5. Despite this, the continuous supply of gas through smooth accretion by gravitational torques 


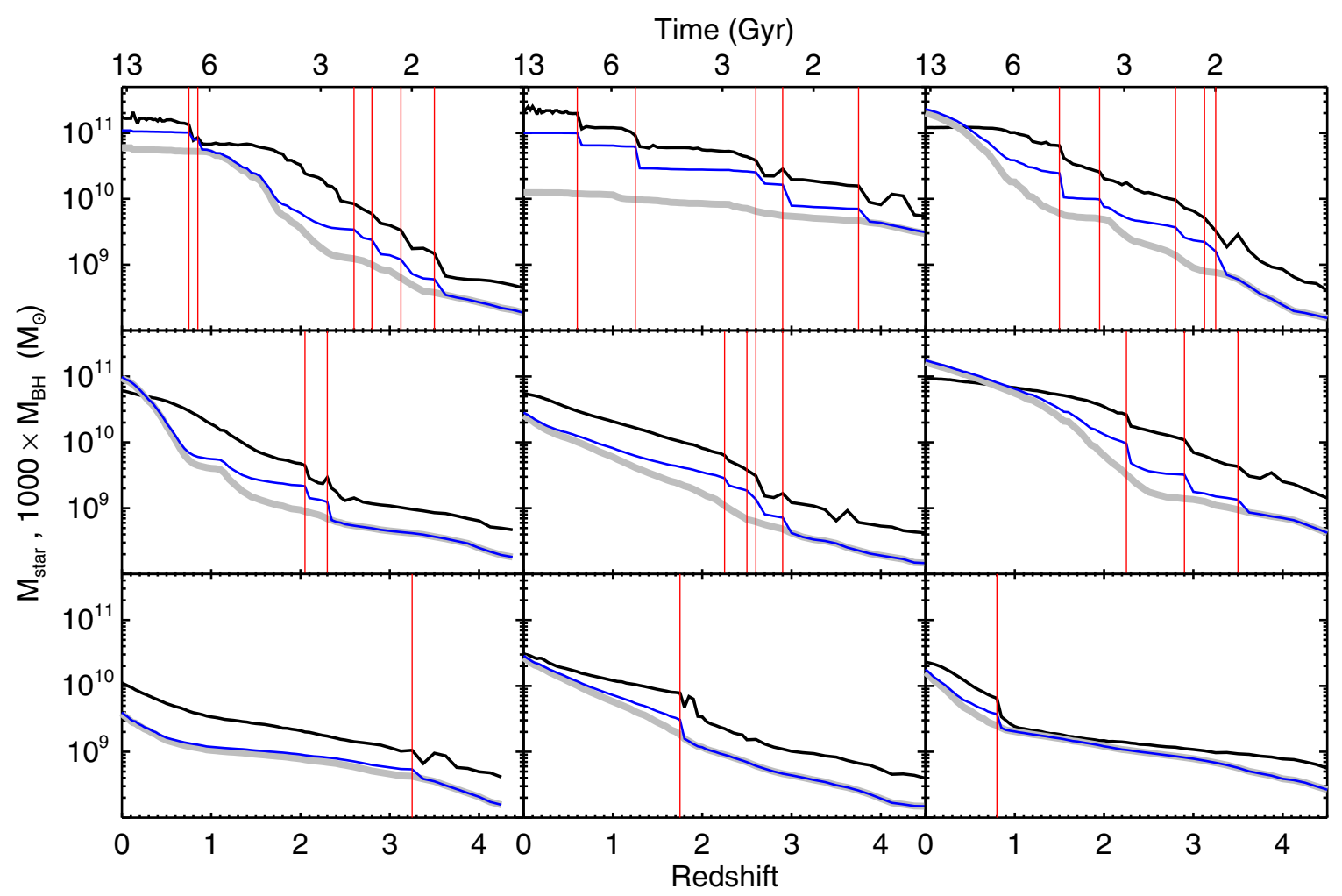

Figure 2. Evolution of the total stellar mass (black lines) and the central black hole mass (blue and gray lines; see below) for nine representative galaxies. Black holes grow according to the gravitational torque rate (Equation (1)) with a mass retention rate $\epsilon_{\mathrm{m}}=0.05$. Initial black hole seeds are taken to be consistent with the $M_{\mathrm{BH}}-M_{\text {bulge }}$ relation (Häring \& Rix 2004) evaluated for the stellar mass within the effective radius of the host galaxy. Black hole mergers are assumed to occur after major galaxy mergers with stellar mass ratios above 1:5, which are indicated by the red vertical lines. Gray lines correspond to black hole growth from torque-limited accretion only (upscaled by a factor of 1000), while blue lines show the total black hole growth including mergers (also upscaled by 1000), where we assume that the merging galaxy has a central black hole with a mass consistent with the corresponding $M_{\mathrm{BH}}-M_{\text {bulge }}$ relation.
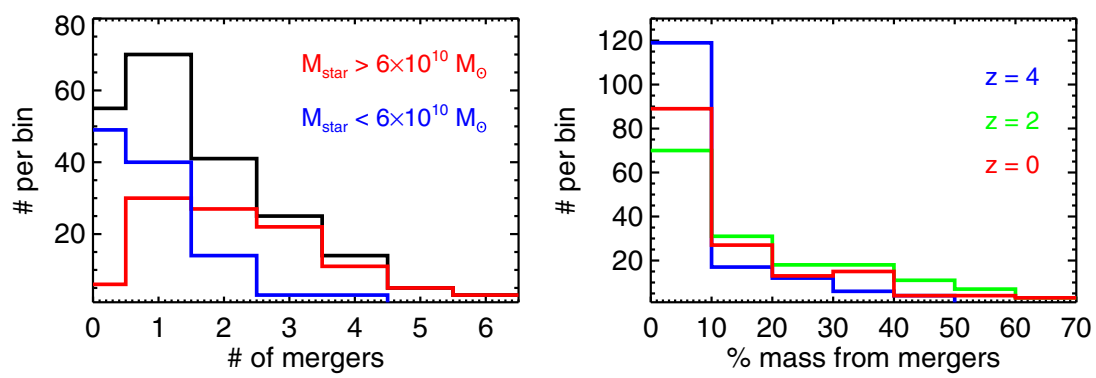

Figure 3. Left: distribution of galaxies in terms of the number of major mergers (with stellar mass ratios $>1: 5$ ) down to $z=0$, for the full galaxy sample (black), for galaxies with stellar masses $M_{\text {star }}>6 \times 10^{10} M_{\odot}$ (red), and for galaxies with stellar masses $M_{\text {star }}<6 \times 10^{10} M_{\odot}$ (blue). Right: distribution of black holes undergoing one or more mergers relative to the percentage of the mass contributed by black hole mergers, computed at $z=0$ (red), $z=2$ (green), and $z=4$ (blue). Black hole seeds and merging black holes are assumed to lie on the $M_{\mathrm{BH}}-M_{\text {bulge }}$ relation for the corresponding host galaxy. Most black holes have a mass contribution of less than $10 \%$ from black hole mergers.

tends to erase the merger histories of black holes. By $z=0$, black hole growth from mergers is typically a small fraction of the total growth, except in some exceptional cases with numerous mergers happening preferentially at low redshift for which the final black hole mass may exceed the total accreted mass by factors of a few (e.g., see the top left and the top middle panels of Figure 2).

This is quantified more rigorously in the right panel of Figure 3, where we show the distribution of black holes in terms of the percentage of mass contributed by mergers, evaluated at three different redshifts. Here, we simply compute the difference between the final black hole mass owing to smooth accretion and mergers and the final black hole mass resulting from smooth accretion alone. For most black holes, the contribution from mergers represents less than $10 \%$ of the total mass, with only a small fraction of black holes having merger contributions $>20 \%$. This occurs despite the fact that the inferred mass fraction from mergers includes some contribution from smooth accretion given by the relatively increased gravitational torque rates for higher mass black holes (Equation (2)). Interestingly, the mass fraction from mergers seems to be higher when evaluated for black holes at $z=2$ relative to either $z=4$ or $z=0$, corresponding to the epoch near the peak of cosmic star formation activity.

Overall, we find that smooth accretion dominates global black hole growth over cosmic time while black hole mergers may represent a non-negligible contribution for the most massive black holes at late times, in agreement with previous studies (e.g., Colberg \& Di Matteo 2008; Dubois et al. 2014; Kulier et al. 2013; Volonteri \& Ciotti 2013). This prediction seems 
robust for the mass range and redshift range that we consider here but may be subject to uncertainties relative to the masses and number densities of seed black holes, the efficiency of black hole merging during galaxy mergers, or the effects of gravitational recoils (e.g., Blecha \& Loeb 2008; Bellovary et al. 2010, 2011; Micic et al. 2011).

\subsection{The $M_{B H}-M_{\text {bulge }}$ Relation}

In Anglés-Alcázar et al. (2013) we showed that black hole growth by gravitational torque-driven accretion yields black holes and host galaxies that evolve on average along the scaling relations from early times down to $z=2$, provided that only a small fraction $\epsilon_{\mathrm{m}}$ of the inflowing gas feeding onto the accretion disk from larger scales is finally accreted by the central black hole. The mass retention rate $\epsilon_{\mathrm{m}} \approx 0.05$ was found to provide the correct normalization over the full redshift range $z=8 \rightarrow 2$, assuming that these black holes follow the local $M_{\mathrm{BH}}-M_{\text {bulge }}$ relation (Häring \& Rix 2004) for the stellar mass within the effective radius.

Figure 4 shows that this result can be extended from the eight zoom disk galaxies in Anglés-Alcázar et al. (2013) to more than 200 galaxies in our cosmological simulation, evolved over a much more extended period of time from $z \sim 4+\rightarrow 0$. Provided that the initial conditions are chosen to agree with the local $M_{\mathrm{BH}}-M_{\text {bulge }}$ relation, black holes and galaxies grow by more than three orders of magnitude in mass approximately along the scaling relation, with no further tuning of the mass retention rate $\epsilon_{\mathrm{m}}$. This conclusion is not affected by the addition of mass growth from black hole mergers, which was neglected in AnglésAlcázar et al. (2013). The top panel of Figure 4 demonstrates this by comparing the evolutionary tracks in the $M_{\mathrm{BH}}-M_{\text {bulge }}$ plane for black holes growing with and without contributions from black hole mergers, shown as the red and blue lines, respectively: the addition of black hole mergers does increase the black hole masses slightly but does not alter the overall trend. Smooth accretion represents most of black hole growth for the majority of the host galaxies and dominates the overall evolution in the $M_{\mathrm{BH}}-M_{\text {bulge }}$ plane, with black hole mergers representing typically a small fraction of the total growth.

Since we do not have an a priori reason to assume that seed black holes correlate with their host galaxy at the starting redshift, we now examine the impact of relaxing this assumption. The middle panel of Figure 4 shows the evolutionary tracks predicted by torque-limited accretion for black hole seeds that are either a factor of 10 above (red) or below (blue) the $M_{\mathrm{BH}}-M_{\text {bulge }}$ relation by the time their host galaxies are first resolved in the simulation. Interestingly, black holes tend to evolve toward the $M_{\mathrm{BH}}-M_{\text {bulge }}$ relation regardless of the initial conditions and with no need for mass averaging through mergers or additional self-regulation processes. This attractor behavior to lie on the $M_{\mathrm{BH}}-M_{\text {bulge }}$ relation was described in AnglésAlcázar et al. (2013) for a small galaxy sample and it is now confirmed for a large number of simulated galaxies. The weak dependence of gravitational torque rates on black hole mass, namely, $\dot{M}_{\text {Torque }} \propto M_{\mathrm{BH}}^{1 / 6}$ (Equation (2)), plays a key role in this overall convergence process, resulting in a rate at which black holes "move" in the logarithmic $M_{\mathrm{BH}}-M_{\text {bulge }}$ plane given by

$$
\frac{d}{d t} \log \left(M_{\mathrm{BH}}\right) \propto \frac{\dot{M}_{\mathrm{BH}}}{M_{\mathrm{BH}}} \propto M_{\mathrm{BH}}^{-5 / 6},
$$

which in turn implies that for a given host galaxy, a lower (higher) mass black hole grows proportionally faster (slower)

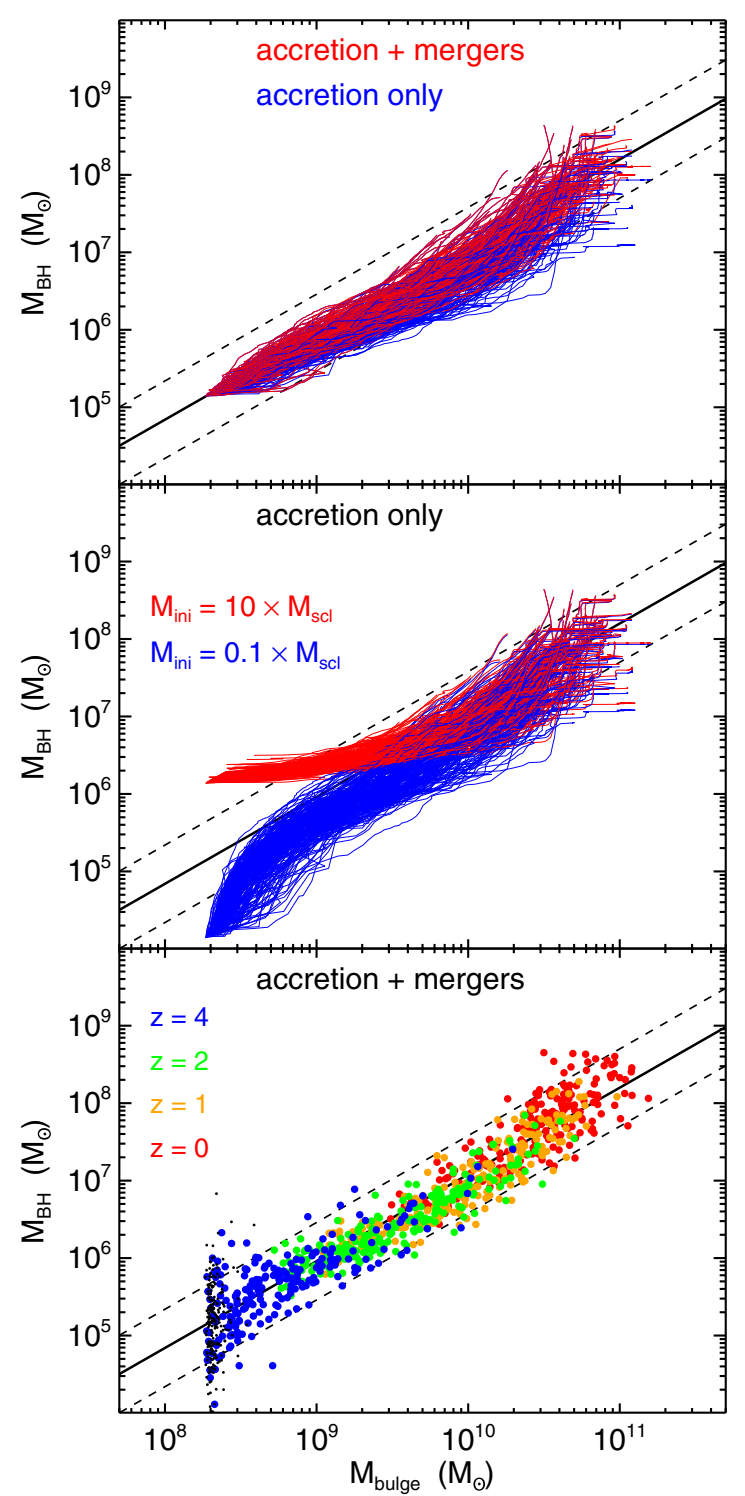

Figure 4. Top: evolutionary tracks of galaxies and central black holes in the $M_{\mathrm{BH}}-M_{\text {bulge }}$ plane for torque-limited growth (blue) and for torque-limited accretion along with mass contributions from black hole mergers (red). Black hole seeds and merging black holes are assumed to lie on the $M_{\mathrm{BH}}-M_{\text {bulge }}$ relation for the corresponding host galaxy. The stellar mass within the effective radius is taken as a proxy for the bulge mass of the host galaxy. The black solid line shows the $M_{\mathrm{BH}}-M_{\text {bulge }}$ relation of Häring \& Rix (2004); black dashed lines indicate a 0.5 dex scatter in black hole mass. Middle: effects of initial conditions on the black hole-galaxy evolutionary tracks. We compute torquelimited growth for seed black holes with initial masses either a factor of 10 above (red) or below (blue) the corresponding $M_{\mathrm{BH}}-M_{\text {bulge }}$ relation. In each case, black holes evolve toward the scaling relation. Bottom: $M_{\mathrm{BH}}-M_{\text {bulge }}$ relation at $z=$ 0 (red), 1 (orange), 2 (green), and 4 (blue) for black holes growing through torque-limited accretion and mergers. Masses of black hole seeds (shown as small black dots) and merging black holes are randomly selected from a lognormal distribution corresponding to the $M_{\mathrm{BH}}-M_{\text {bulge relation for the appropriate }}$ galaxy and time step, assuming a 0.5 dex scatter in black hole mass.

relative to a black hole lying on the $M_{\mathrm{BH}}-M_{\text {bulge }}$ relation. We will explore this attractor behavior in more detail in Section 3.6.

Given that black holes tend to evolve onto the $M_{\mathrm{BH}}-M_{\text {bulge }}$ relation, it seems justified to adopt the simplification that black holes and galaxies are already on the scaling relation by the time we define the initial conditions, i.e., when the host galaxy is first resolved in the cosmological simulation. Nonetheless, there is significant scatter in black hole mass at a given bulge 
mass despite the overall convergence toward the $M_{\mathrm{BH}}-M_{\text {bulge }}$ relation. This intrinsic scatter does not go away with subsequent evolution; therefore, it should be taken into account when defining the initial conditions for black hole growth as well as the mass contribution from black hole mergers.

The bottom panel of Figure 4 shows the $M_{\mathrm{BH}}-M_{\text {bulge }}$ relation obtained at different redshifts when black hole seeds and merging black holes are randomly chosen from a log-normal distribution corresponding to the $M_{\mathrm{BH}}-M_{\text {bulge }}$ relation for the appropriate galaxy and time step, but assuming a $0.5 \mathrm{dex}$ scatter in black hole mass. Overall, our full model for torquelimited growth is consistent with a close-to-linear non-evolving $M_{\mathrm{BH}}-M_{\text {bulge }}$ relation, so long as the initial conditions at some reference redshift are not biased toward either higher-mass or lower-mass black holes relative to their host galaxies. Note that some initially large log-normal scatter may produce a bias toward higher-mass black holes at later times because their timescale for convergence toward the scaling relation is significantly longer relative to lower-mass black holes. We explore this in Section 3.6.

Black hole mergers may reduce the scatter of the $M_{\mathrm{BH}}-M_{\text {bulge }}$ relation by recurrent mass averaging (Hirschmann et al. 2010), a process that has indeed been suggested as the actual physical mechanism giving rise to the black hole-galaxy scaling relations (Peng 2007; Jahnke \& Macciò 2011). Mergers actually seem to reduce the scatter somewhat (Figure 4, top panel), but major mergers $(>1: 5)$ are clearly not frequent enough for our Galaxy sample to establish the $M_{\mathrm{BH}}-M_{\text {bulge }}$ relation in the first place. In some cases, the merging of several slightly over-massive black holes may yield outliers in the $M_{\mathrm{BH}}-M_{\text {bulge }}$ relation even under normal accreting conditions. It is, nonetheless, challenging to explain recent observations in the local universe suggesting the presence of highly over-massive black holes compared to their host galaxies (Bogdán et al. 2012; van den Bosch et al. 2012; but see Emsellem 2013). In the context of torque-limited growth, it is plausible that such extreme objects could form from highly above-average accreting conditions, such as a favorably oriented galaxy merger.

Observations are currently inconclusive regarding the slope and normalization of the scaling relations at high redshift. While several studies have reported an increase in the black hole mass to host galaxy mass ratio for individual systems at higher redshifts (Treu et al. 2007; Decarli et al. 2010; Greene et al. 2010; Merloni et al. 2010; Bennert et al. 2011; Targett et al. 2012) there remain significant concerns about to systematics in the mass estimators (e.g., Park et al. 2013) and biases introduced by selection effects (Lauer et al. 2007; Shen \& Kelly 2010; Schulze \& Wisotzki 2011). Indeed, a number of observations seem consistent with little or no evolution in the black hole mass to host galaxy mass ratio (Jahnke et al. 2009; Cisternas et al. 2011a; Schramm \& Silverman 2013). Assuming a non-evolving mass retention rate $\left(\epsilon_{\mathrm{m}}\right)$ in the accretion flow, torque-limited growth predicts no significant evolution of the $M_{\mathrm{BH}}-M_{\text {bulge }}$ relation unless the initial conditions are substantially different relative the local scaling relation. In Section 3.6, we evaluate the characteristic time scales for convergence toward the $M_{\mathrm{BH}}-M_{\text {bulge }}$ relation.

Note that we have not attempted to estimate the "true" bulge mass in analogy with observations, but instead replaced it in the $M_{\mathrm{BH}}-M_{\text {bulge }}$ relation by the stellar mass within the effective radius of the host galaxy. Torque-limited growth yields a correlation between black hole mass and stellar mass regardless of the morphology of the galaxy. This suggests that the processes driving the morphological evolution of the stellar component in galaxies may not be fundamental for the growth of their central black hole (Marleau et al. 2013; Simmons et al. 2013). Incidentally, there is increasing evidence for significant black hole growth taking place in disk dominated galaxies with no merger signatures (Gabor et al. 2009; Georgakakis et al. 2009; Cisternas et al. 2011b; Kocevski et al. 2012; Mullaney et al. 2012b; Schawinski et al. 2012; Treister et al. 2012), while both, galaxy mergers and secular evolution, are commonly invoked as primary mechanisms for bulge formation (e.g., Hopkins et al. 2010; Kormendy \& Ho 2013).

This simple scenario of black hole-galaxy coevolution is challenged by observations in the local universe suggesting that black holes correlate differently with different galaxy components (Graham 2008; Hu 2008; Graham et al. 2011; Kormendy et al. 2011; Kormendy \& Ho 2013). Recent results imply a broken power-law relation between the masses of black holes and their host spheroids (Graham 2012; Graham \& Scott 2013; Scott et al. 2013), with lower-mass black holes in Sérsic galaxies $\left(M_{\mathrm{BH}} \lesssim 10^{8} M_{\odot}\right)$ following a steeper relation $M_{\mathrm{BH}} \propto M_{\text {bulge }}^{2}$ below the classic nearly linear scaling. While our simulations lack the resolution required for a detailed analysis of the $z=0$ $M_{\mathrm{BH}}-M_{\text {bulge }}$ relation and its morphological dependence, we note that torque-limited growth yields a qualitatively similar steep trend for initially under-massive black holes as they evolve onto the $M_{\mathrm{BH}}-M_{\text {bulge }}$ relation (Figure 4, middle panel). Observations of black holes in low-mass galaxies may thus provide significant constraints on the initial conditions for massive black hole growth (Greene 2012).

\subsection{Evolution of Eddington Ratios}

Gravitational torques drive gas inflows from galactic scales down to sub-parsec scales, feeding the accretion flow near the black hole, and governing the co-evolution of black holes and galaxies. The observed black hole-galaxy scaling relations are a natural outcome of this process. In this section, we explore the accretion histories resulting from torque-limited growth as well as implications for observations of active systems across cosmic time.

The left panel of Figure 5 shows the evolution of Eddington ratios with redshift, defined here as the black hole accretion rate in units of Eddington, $\lambda \equiv \dot{M}_{\mathrm{BH}} / \dot{M}_{\text {edd }}$. For all black holes, $\dot{M}_{\mathrm{BH}}$ is calculated from Equation (1) for the mass retention rate $\epsilon_{\mathrm{m}}=0.05$. The Eddington rate is given by the usual definition, $\dot{M}_{\text {edd }}=4 \pi G M_{\mathrm{BH}} m_{\mathrm{p}} /\left(\eta \sigma_{\mathrm{T}} c\right)$, where the accretion efficiency, $\eta$, represents the maximum amount of potential energy per unit rest mass energy that can be extracted from the innermost stable circular orbit of the accretion disk around the black hole. Throughout this paper, we adopt a fixed value $\eta=0.1$ (e.g., Yu $\&$ Tremaine 2002; Marconi et al. 2004) and ignore its intrinsic dependence on black hole spin.

Gray lines in Figure 5 (left panel) correspond to the accretion histories of individual black holes. Despite our limited time resolution, restricted by the number of output files produced during the simulation, accretion rates show significant variability relative to cosmological timescales. This variability follows from the complex evolution of the inner regions of galaxies (Hopkins \& Quataert 2010), which manifests itself in the gravitational torque model as significant variations in morphological properties within the radial aperture $R_{0}$ (Hopkins \& Quataert 2011). Black points with error bars show median Eddington ratios within logarithmically spaced bins in $1+z$ and the 5 and 

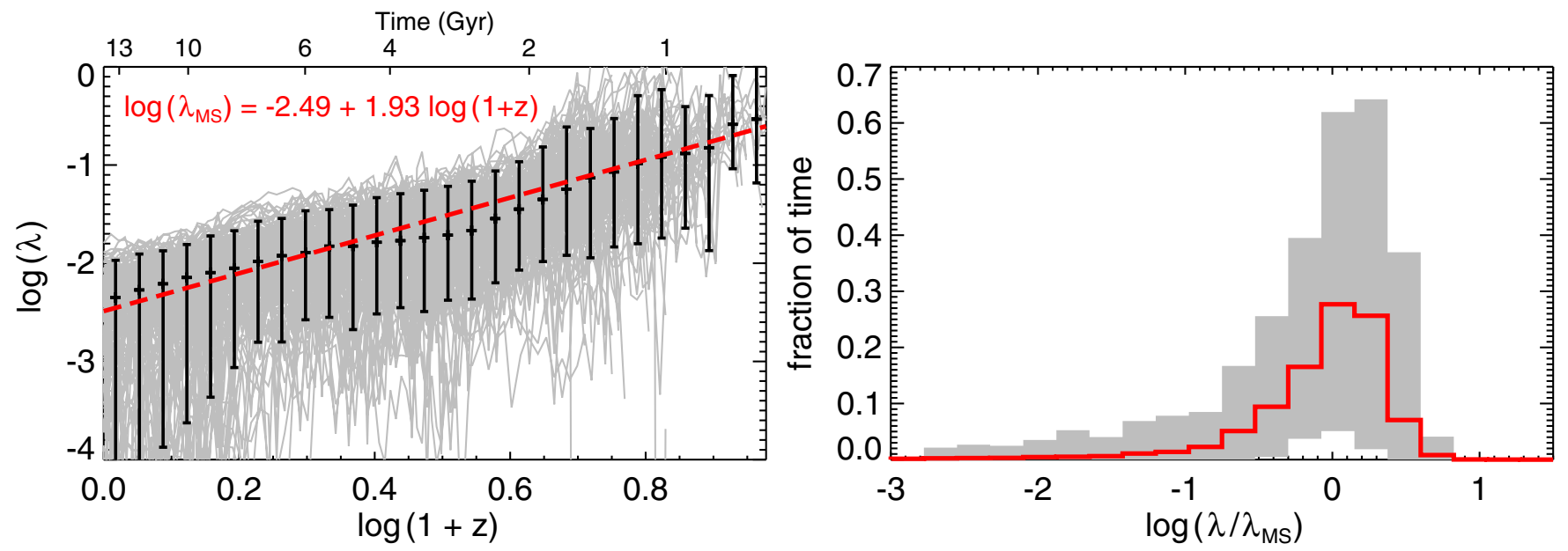

Figure 5. Left: Eddington ratios, $\lambda \equiv \dot{M}_{\mathrm{BH}} / \dot{M}_{\text {Edd }}$, as a function of redshift for the central black holes of each of the 213 galaxies selected at $z=0$. Gray lines show individual accretion histories while black points with error bars show median values within bins logarithmically spaced in $(1+z)$ and the corresponding 5 and 95 percentiles of the distribution. Accretion rates are calculated according to Equations (1) and (2) for a mass retention rate $\epsilon_{\mathrm{m}}=0.05$. The red dashed line shows the best power law fit to the median values: $\log \left(\lambda_{\mathrm{MS}}\right) \approx-2.49+1.93 \log (1+z)$. Right: fraction of the evolution time down to $z=0$ that black holes spend accreting at a given Eddington ratio relative to $\lambda_{\mathrm{MS}}(z)$. The red solid line shows the time spent in a given $\lambda / \lambda_{\mathrm{MS}}$ bin averaged over all black holes and the gray shaded region indicates the 5 and 95 percentiles of the distribution of time fractions in each $\lambda / \lambda_{\mathrm{MS}}$ bin.

95 percentiles of the distribution, indicating that there is also a significant scatter for our sample of black holes at any given redshift.

Despite the large scatter, our simulations reveal a common trend for the evolution of Eddington ratios. Black holes are typically accreting at high Eddington ratios at early times, with median values $\lambda>10 \%$ at $z \gtrsim 6$ and may even exceed the Eddington limit in some cases. At lower redshifts, a gradual decrease in Eddington ratios yields $\lambda \sim 1-10 \%$ at $z \approx 2$ (as previously found in Anglés-Alcázar et al. 2013), reaching typical present day values $\lambda \sim 0.1 \%-1 \%$ at $z=0$. As shown by the red dashed line in the left panel of Figure 5, a simple power law provides a good fit to the redshift dependence of the median Eddington ratio, albeit with significant scatter:

$$
\log \left(\lambda_{\mathrm{MS}}\right) \approx-2.49+1.93 \log (1+z)
$$

where we have ignored any intrinsic dependence of Eddington ratios on black hole mass (see below). The exact slope and normalization in Equation (7) are somewhat dependent on sample selection and initial conditions (Section 3.6). Nonetheless, this relation provides a useful tool for characterizing black hole accretion histories, in analogy with the star formation main sequence, which can be defined in terms of the median specific SFR for a given redshift interval (e.g., Davé et al. 2011b; Elbaz et al. 2011).

We can now evaluate the evolution of Eddington ratios relative to the sequence defined by Equation (7). The right panel of Figure 5 shows the fraction of time that black holes spend accreting at a given Eddington ratio in units of the median value $\lambda_{\text {MS }}$. For each black hole at a given redshift, we calculate the ratio $\lambda(z) / \lambda_{\mathrm{MS}}(z)$ to which we assign the duration of the current time step. Then, by adding up the contributions from all time steps, we estimate the fraction of the total evolution time (down to $z=0$ ) during which a given black hole grows at some Eddington ratio relative to the main sequence value. We indicate as the red solid line the average fraction of time spent in a given $\lambda / \lambda_{\mathrm{MS}}$ bin over all black holes (equivalent to the probability per logarithmic interval), while the gray shaded region corresponds to the 5 and 95 percentiles of the distribution in each $\lambda / \lambda_{\mathrm{MS}}$ bin.
The right panel of Figure 5 shows that black holes spend most of their time accreting near the median Eddington ratio for the whole population, suggesting that Equation (7) may, indeed, represent an "AGN main sequence" (Mullaney et al. 2012a). Eddington ratios can be roughly described by a lognormal distribution centered at $\lambda_{\mathrm{MS}}(z)$ at all redshifts, but note the asymmetry with respect to $\lambda=\lambda_{\mathrm{MS}}$, with a relative increased probability for black holes accreting at lower Eddington ratios (especially at low redshift). One caveat here is the limited time resolution; our inferred Eddington ratios correspond to average values within time intervals ranging from $\sim 10$ to $300 \mathrm{Myr}$ in the redshift range $z \sim 6 \rightarrow 0$, while AGN luminosities exhibit strong variability over a large dynamic range, from hours (e.g., McHardy 2013) to Myr timescales (e.g., McNamara \& Nulsen 2007; Gonçalves et al. 2008). Thus, the right panel of Figure 5 corresponds to departures from the AGN main sequence $\left(\lambda_{\mathrm{MS}}\right)$ on timescales comparable to typical galaxy dynamical timescales. Shorter timescale variability that we cannot track may have important consequences for the observed distribution of Eddington ratios and the inferred connection between star formation and AGN activity (Hickox et al. 2014).

\subsection{Bolometric Luminosities}

The radiative properties of accretion flows around AGNs are thought to depend primarily on the mass inflow rate onto the black hole, with a relatively well defined transition between radiatively efficient and radiatively inefficient modes at Eddington ratios of about a few percent (Narayan \& Yi 1995b; Maccarone et al. 2003; Greene et al. 2006), in close analogy to Galactic stellar-mass black holes in X-ray binaries (Remillard \& McClintock 2006). Here, we infer AGN bolometric luminosities by assuming that there is an accretion state transition at $\lambda_{\text {crit }}=0.03$, as in Merloni \& Heinz (2008). For the radiatively efficient mode $\left(\lambda>\lambda_{\text {crit }}\right)$, the bolometric luminosity is simply proportional to the accretion rate, $L_{\text {bol }}=\eta \dot{M}_{\mathrm{BH}} c^{2}$, and, therefore, $L_{\text {bol }} / L_{\text {edd }}=\lambda$. For radiatively inefficient accretion flows $\left(\lambda<\lambda_{\text {crit }}\right)$ we compute $L_{\text {bol }}=\eta \dot{M}_{\mathrm{BH}} c^{2}\left(\lambda / \lambda_{\text {crit }}\right)$.

Figure 6 shows the ratio of the bolometric luminosity to the Eddington luminosity, $L_{\mathrm{bol}} / L_{\mathrm{edd}}$, as a function of black hole mass, evaluated at four different redshifts. With the definition of 


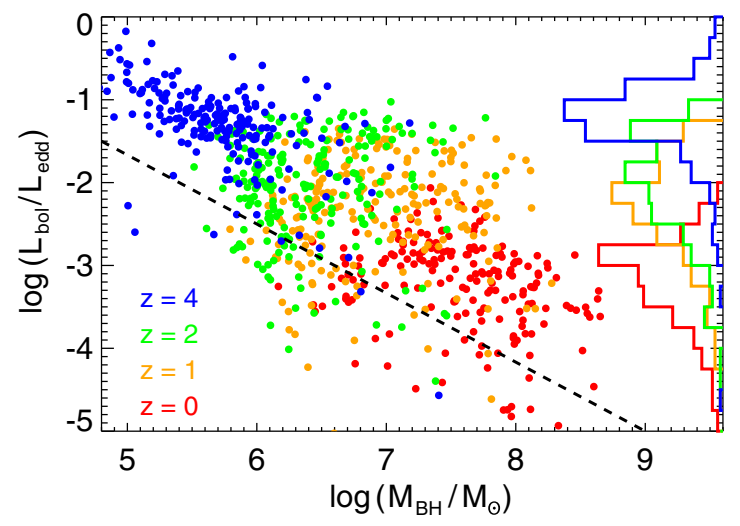

Figure 6. Bolometric luminosity in units of the Eddington luminosity, $L_{\text {bol }} / L_{\text {edd }}$, as a function of black hole mass for our sample of 213 black holes at $z=0$ (red), 1 (orange), 2 (green), and 4 (blue). Bolometric luminosities are calculated from accretion rates by assuming that there is a transition between radiatively efficient to radiatively inefficient accretion at $\lambda_{\text {crit }}=0.03$ (Merloni \& Heinz 2008). Histograms show the distribution of Eddington ratios at each redshift (arbitrarily normalized). The black dashed line corresponds to the scaling $\lambda \propto M_{\mathrm{BH}}^{-5 / 6}$.

$\lambda_{\text {crit }}$ adopted here, the shift from radiatively efficient accretion to radiatively inefficient accretion occurs at $z \approx 1-2$ for the median black hole. Thus, the probability of a galaxy hosting an AGN in the radiatively inefficient mode increases at lower redshifts. The inferred bolometric luminosity corresponding to a given accretion rate is lower for black holes growing in the radiatively inefficient mode. The ratio $L_{\text {bol }} / L_{\text {edd }}$ is, therefore, characterized by a stronger evolution with redshift relative to the intrinsic accretion rate $(\lambda)$, decreasing by about three orders of magnitude from $z=4$ to $z=0$.

At a fixed redshift, the inferred $L_{\text {bol }} / L_{\text {edd }}$ values roughly follow a log-normal distribution, as expected from Figure 5. At $z=4$, most black holes radiate in a relatively narrow log-normal distribution around $L_{\text {bol }} / L_{\text {edd }} \approx 0.05$, right above $\lambda_{\text {crit }}$. At lower redshifts, the width of the $L_{\text {bol }} / L_{\text {edd }}$ distribution increases, with an extended tail toward low luminosities. This is particularly evident at $z \approx 1-2$ when most black holes are undergoing a transition from radiatively efficient $\left(\lambda>\lambda_{\text {crit }}\right)$ to radiatively inefficient $\left(\lambda<\lambda_{\text {crit }}\right)$ accretion. Note that the absence of black holes with masses $>10^{8} M_{\odot}$ at $z \sim 2$ simply reflects the lack of massive galaxies early enough to host such massive black holes in our simulated volume.

Figure 6 shows an anti-correlation between $L_{\text {bol }} / L_{\text {edd }}$ and black hole mass. For a given host galaxy, the gravitational torque rate scaled by the Eddington rate is lower for highermass black holes, $\lambda \propto M_{\mathrm{BH}}^{-5 / 6}$. This should imply a strong trend of decreasing Eddington ratios for increasing black hole mass at a fixed redshift; this trend is, however, weaker than expected for our sample of black holes or even nonexistent at $z \approx 1-2$, suggesting a complex evolution of black hole accretion besides the intrinsic dependence on $M_{\mathrm{BH}}$. Indeed, we will show in Section 4.1 (Figure 12) that higher mass galaxies are more compact than lower mass galaxies while having a similar disk fraction; this results in higher values of $R_{0}^{-3 / 2} M_{\mathrm{d}}$ that partially compensate for the decrease in $\lambda$ values with increasing $M_{\mathrm{BH}}$. The net effect is a $\lambda\left(M_{\mathrm{BH}}\right)$ dependence that is weaker than the a priori expected $\lambda \propto M_{\mathrm{BH}}^{-5 / 6}$, which may only be identified for a black hole sample spanning a sufficient mass range.

Selection effects may have a significant impact on the observed evolution of Eddington ratios and their dependence on black hole mass. This is illustrated in Figure 7 where we calculate the median bolometric luminosity in Eddington units $\left(L_{\text {bol }} / L_{\text {edd }}\right)$ as a function of redshift $(z=2 \rightarrow 0)$ for two sub-samples of black holes: those with masses above and below $M_{\mathrm{BH}}=2 \times 10^{7} M_{\odot}$. The left panel of Figure 7, which includes all of our 213 black holes selected at $z=$ 0 , shows no clear separation of the high-mass and low-mass sub-samples in terms of their median $L_{\text {bol }} / L_{\text {edd }}$. The high-mass sample is characterized by larger scatter at all redshifts, perhaps suggesting a stronger variability, but the limited mass range does not allow us to discern an intrinsic decrease in $\lambda$ with $M_{\mathrm{BH}}$.

The right panel of Figure 7 mimics the effects of observational sensitivity limits by calculating median Eddington ratios for the two sub-samples but now only including black holes radiating with a total "observed" flux higher than $F_{\text {lim }}=3 \times 10^{-15} \mathrm{erg} \mathrm{s}^{-1} \mathrm{~cm}^{-2}$, equivalent to a bolometric luminosity $L_{\text {bol }} \approx 10^{44} \mathrm{erg} \mathrm{s}^{-1}$ at $z=2$. For each black hole we calculate the total flux from the bolometric luminosity as $F=L_{\text {bol }} /\left(4 \pi d_{\mathrm{L}}^{2}\right)$, where $d_{\mathrm{L}}$ is the luminosity distance at the corresponding redshift. Our flux-limited sample of black holes shows a clear dependence of Eddington ratios on black hole mass for the full redshift range $z=2 \rightarrow 0$, with the higher mass sample dominating the low $L_{\text {bol }} / L_{\text {edd }}$ regime. This simple experiment illustrates how the inferred evolution of black hole populations can be affected by sensitivity limits, even neglecting obscuration effects. Black holes growing at low Eddington ratios may be missed in flux-limited surveys preferentially at higher redshifts and for lower-mass black holes, as explicitly shown by Kollmeier et al. (2006). This may result in (1) a stronger apparent evolution of Eddington ratios with redshift and (2) an artificially increased systematic offset between the typical Eddington ratios of higher-mass and lower-mass black holes.

A direct comparison of the evolution of Eddington ratios with observations is not trivial given that differences in, for example, selection techniques and completeness limits often yield contrasting results among AGN populations. Despite this, a wide range of observations indicate that the typical Eddington ratios of accreting black holes increase at higher redshifts (Kollmeier et al. 2006; Netzer \& Trakhtenbrot 2007; Kauffmann \& Heckman 2009; De Rosa et al. 2011; Trakhtenbrot et al. 2011; Aird et al. 2012; Bongiorno et al. 2012; Lusso et al. 2012; Trakhtenbrot \& Netzer 2012), in broad agreement with our results and consistent with expectations from AGN synthesis models (e.g., Merloni \& Heinz 2008; Shankar et al. 2013). However, the typical median values may vary significantly for different AGN populations even at similar redshifts, e.g., ranging from near Eddington accretion for the AGN sample of Kollmeier et al. (2006) at $z \sim 0.3-4$ to sub-Eddington growth for the AGN population studied by Lusso et al. (2012) at $z \sim 1-2$. Indeed, Trump et al. (2011) reported the presence of two separate populations in X-ray-selected AGNs, associated with black holes accreting in radiatively efficient $(\lambda \gtrsim 0.01)$ and radiatively inefficient $(\lambda \lesssim 0.01)$ modes. For torque-limited growth, such a transition between accretion modes occurs multiple times for typical black hole accretion histories, but with an increased probability for radiatively inefficient accretion at lower redshifts.

The situation is less clear with respect to the distribution of Eddington ratios and its dependence on black hole mass. Numerous observational studies have reported Eddington ratio distributions consistent with a log-normal distribution for a wide range of redshifts (Kollmeier et al. 2006; Netzer \& Trakhtenbrot 

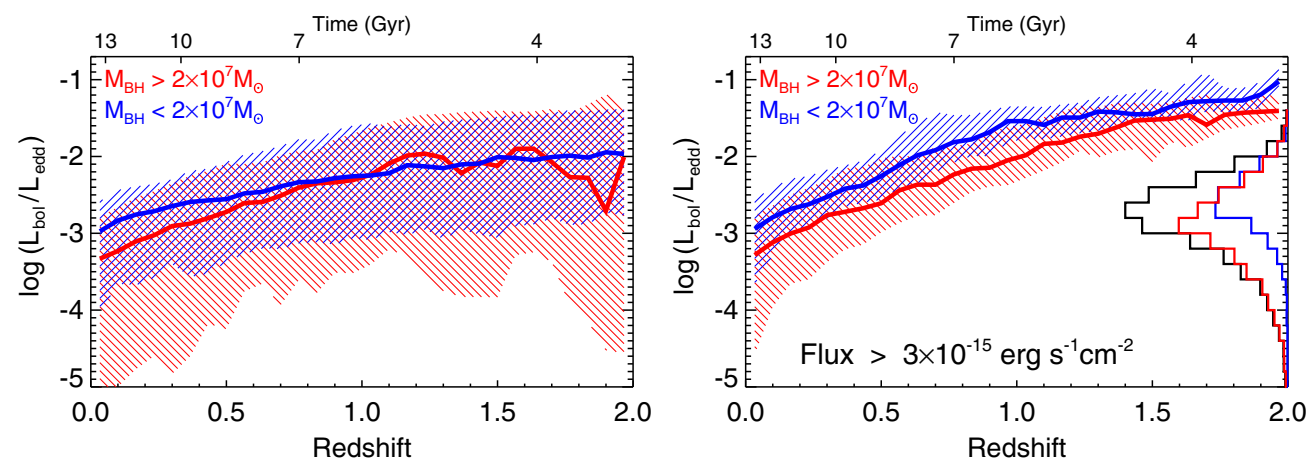

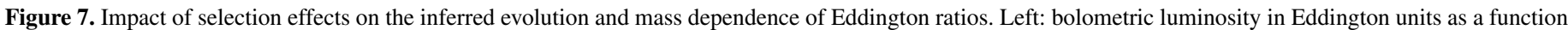

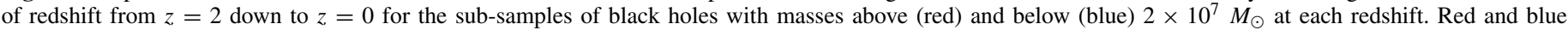

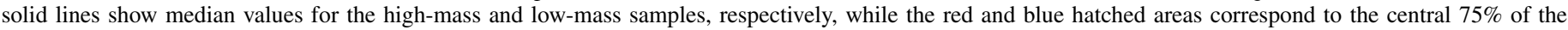

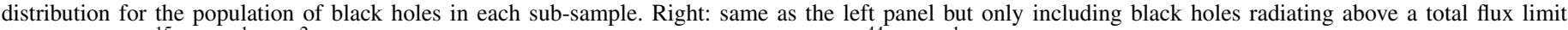

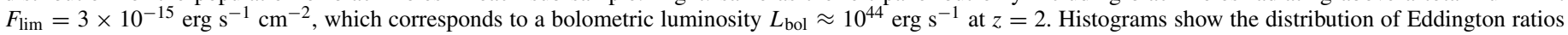
(arbitrarily normalized) for all black holes below $z<0.5$ (black) and for the high-mass (red) and low-mass (blue) sub-samples.

2007; Hickox et al. 2009; Netzer 2009; Trakhtenbrot et al. 2011; Trump et al. 2011; Lusso et al. 2012) in agreement with our results, while other studies favor a universal power law distribution function independent of black hole mass (Aird et al. 2012; Bongiorno et al. 2012). Trakhtenbrot \& Netzer (2012) reported a significant decrease in Eddington ratios with increasing black hole mass in the redshift range $z=0-2$, while Kelly $\&$ Shen (2013) found that $\lambda$ is approximately independent of black hole mass at low $(z<0.8)$ and high $(z>2.65)$ redshifts but increases with black hole mass at intermediate redshifts. Kollmeier et al. (2006) examined the distribution of Eddington ratios in the redshift range $z \sim 0.3-4$ and found a characteristic log-normal distribution independent of black hole mass and redshift down to well-characterized completeness limits. At low redshift, Kauffmann \& Heckman (2009) identified two distinct distributions of Eddington ratios: black holes in star forming galaxies follow a log-normal distribution that only weakly depends on the black hole mass and black holes in passive galaxies follow a power-law distribution function with a normalization that strongly depends on the black hole mass.

We find that Eddington ratios averaged over galaxy evolution timescales can be roughly described by a lognormal distribution with increasing width at lower redshifts and with a median value evolving as a power law in $(1+z)$ broadly similar to the cosmic evolution of specific SFRs. Furthermore, the combined dependence of accretion rates on black hole mass and galaxy surface density $\left(R_{0}^{-3 / 2} M_{\mathrm{d}}\right)$ yields a weak trend of decreasing median Eddington ratios with increasing black hole mass at all redshifts. Encouragingly, similar trends have been identified by Shankar et al. (2013) as key elements in reproducing a number of observations, including the observed Eddington ratio distributions, the high AGN fractions at low redshift, and the higher frequency of AGNs in higher-mass galaxies.

\subsection{The SFR-AGN Connection}

Torque-limited accretion yields black holes growing, on average, in tandem with their host galaxies (Figure 4). Smooth accretion dominates the total growth of black holes (Figure 3) and their host galaxies (e.g., Murali et al. 2002; Keres et al. 2005), implying that there must be some connection between the total SFR of galaxies and their nuclear activity on cosmological timescales. In this section, we extend our current analysis to a significantly larger number of black holes and host galaxies to present predictions for the relation between galaxy SFRs and AGN activity for an increased dynamic range.
For this purpose, we select all galaxies with stellar masses $M_{\text {star }}>10^{8} M_{\odot}$ from our $\left[32 h^{-1} \mathrm{Mpc}\right]^{3}$ simulation volume at different redshifts, for example there is a total of 4356 and 5815 galaxies at $z=0$ and $z=2$, respectively. At each redshift, we assign central black holes to every galaxy by assuming consistency with the local $M_{\mathrm{BH}}-M_{\text {bulge }}$ relation (Häring \& Rix 2004); black holes are randomly selected from a log-normal distribution centered on the $M_{\mathrm{BH}}-M_{\text {bulge }}$ relation for each galaxy and assuming a 0.5 dex scatter in black hole mass. We estimate accretion rates by direct evaluation of Equation (1) with $\epsilon_{\mathrm{m}}=0.05$, where we now employ a radial aperture equal to the effective radius of the host galaxy, $R_{0}=R_{\text {eff }}$ (see Equation (2)). This allows us to evaluate accretion rates for virtually all galaxies within our simulation volume. The effects of using different radial apertures in the gravitational torque model are discussed in Section 4.

Figure 8 shows the SFR $-\dot{M}_{\mathrm{BH}}$ relation predicted by the gravitational torque model. Points with different colors represent the location of individual systems in the SFR- $\dot{M}_{\mathrm{BH}}$ plane at different redshifts, from $z=4$ (blue) to $z=0$ (red). Despite the large scatter, the increased dynamic range allows us to identify a clear relation extending over a few orders of magnitude in both SFR and $\dot{M}_{\mathrm{BH}}$; the inferred slope resembles the closeto-linear scaling expected from the local $M_{\mathrm{BH}}-M_{\text {bulge }}$ relation. Note that the overall normalization of the SFR relative to $\dot{M}_{\mathrm{BH}}$ is controlled by the mass retention rate, which is set to $\epsilon_{\mathrm{m}}=0.05$ to match the $M_{\mathrm{BH}}-M_{\text {bulge }}$ relation of Häring \& Rix (2004) for the stellar mass within the effective radius of the host galaxy (i.e., for one-half of the total stellar mass).

Torque-limited growth yields, therefore, a connection between AGN activity and SFR on timescales comparable to the dynamical timescale of galaxies. Cosmological gas infall and transport of angular momentum in the galaxy by gravitational instabilities appear to be the primary physical drivers behind this relation. The evolution of specific SFRs with redshift can be roughly described by a characteristic power law given by the evolution of the gas accretion rate onto dark matter halos, $\sim(1+z)^{2.25}$ (Dekel et al. 2009); feedback can modify this by suppressing star formation at early epochs and providing recycling wind material back onto galaxies at later times, yielding a shallower evolution of the specific SFR with redshift (Oppenheimer et al. 2010; Davé et al. 2011b; Anglés-Alcázar et al. 2014). Intriguingly, gravitational torques provide gas inflows for fuelling the central AGN at a roughly constant fraction of the SFR in galaxies, in a time averaged sense. 


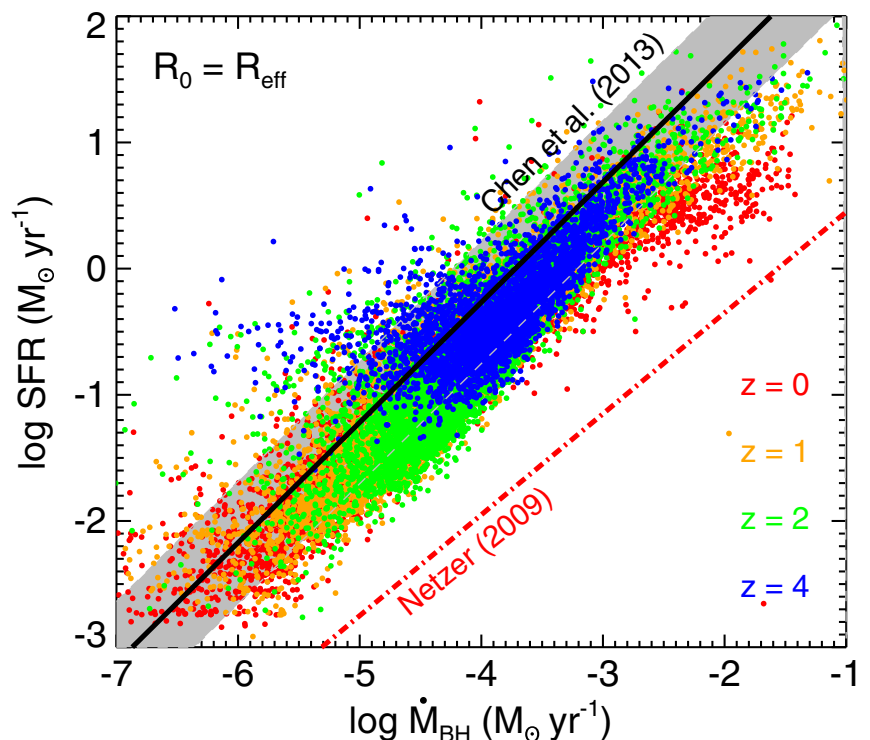

Figure 8. Total SFR as a function of central black hole accretion rate for all galaxies with stellar masses $M_{\text {star }}>10^{8} M_{\odot}$ found within our $\left[32 h^{-1} \mathrm{Mpc}\right]^{3}$ simulation volume at $z=0$ (red), 1 (orange), 2 (green), and 4 (blue). Accretion rates are calculated by direct evaluation of Equation (1) (with $\epsilon_{\mathrm{m}}=0.05$ ), assuming that black holes lie on the $M_{\mathrm{BH}}-M_{\text {bulge }}$ relation (Häring \& Rix 2004 , for the stellar mass within the effective radius) with a 0.5 dex scatter in black hole mass at all redshifts. Torque-limited inflow rates (Equation (2)) are calculated from host galaxy properties evaluated within a radial aperture equal to the effective radius, $R_{0}=R_{\text {eff }}$. The black solid line shows the SFR $-M_{\mathrm{BH}}$ correlation reported by Chen et al. (2013) for star-forming galaxies in the redshift range $0.25<z<0.8$, and the gray shaded region corresponds to their estimated uncertainty in the normalization. The red dot-dashed line shows the $L_{\mathrm{SF}} \propto L_{\mathrm{AGN}}^{0.8}$ relation of Netzer (2009) for low redshift AGN-dominated systems.

The evolution of Eddington ratios resulting from this process can also be described as a power law in $1+z$ (Figure 5), suggesting that central black holes evolve along an AGN main sequence similar to the main sequence for star-forming galaxies (Mullaney et al. 2012a). Note that this is in contrast to recent interpretations of the SFR-AGN connection in terms of positive AGN feedback triggering star formation (e.g., Silk 2013; Zubovas et al. 2013).

The SFR $-\dot{M}_{\mathrm{BH}}$ connection is, however, not direct on a galaxyby-galaxy basis at all times. Even at the scales resolved here, the accretion rates are highly variable (Figure 5) and may experience significant variations uncorrelated with the host galaxy SFR (Hopkins \& Quataert 2010, 2011; Anglés-Alcázar et al. 2013). Indeed, the evolutionary tracks of individual systems in the SFR $-\dot{M}_{\mathrm{BH}}$ plane are rather complicated. In our simulations, star formation responds to variations in gas surface density via a sub-grid prescription tuned to match the observed Kennicutt (1998) relation (Springel \& Hernquist 2003). On average, gas inflows by gravitational torques also increase with gas surface density via the $R_{0}^{-3 / 2} M_{\mathrm{d}}$ term in Equation (2) (as well as the intrinsic dependence on $f_{\text {gas }}$ ), but critically depend on the overall morphology of the inner region of the galaxy. Variations in the fraction of mass in a disk component $\left(f_{\mathrm{d}}\right)$ are responsible for significant scatter in the SFR $-M_{\mathrm{BH}}$ relation. Note that we have assumed a fixed mass retention rate $\epsilon=0.05$; relaxing this assumption could result in additional scatter.

Do we observe such a correlation between SFR and AGN activity? Figure 8 shows substantial agreement between our results and recent observations by Chen et al. (2013). These authors showed that the average central black hole accretion rate for star-forming galaxies in the redshift range $0.25<z<0.8$ is almost linearly proportional to the SFR; their inferred SFR $-\dot{M}_{\mathrm{BH}}$ relation is shown as the black solid line, and the uncertainty in the normalization is indicated by the gray shaded region. A roughly similar correlation between star formation and AGN luminosity was reported by Netzer (2009) for active galaxies at lower redshifts, $L_{\mathrm{SF}} \propto L_{\mathrm{AGN}}^{0.8}$, which we show as the red dot-dashed line in Figure 8. In this case, the normalization is significantly lower, perhaps unsurprisingly given that this relation was found for AGN-dominated systems. Note that we are extending the correlations of Netzer (2009) and Chen et al. (2013) to an SFR $-\dot{M}_{\mathrm{BH}}$ regime well below their detection limits. We predict that similar correlations should continue down to significantly lower levels of star formation and black hole accretion.

Positive correlations between average SFR and AGN luminosity have also been reported by a number of authors at higher redshifts (Feltre et al. 2013), in general agreement with our findings, but seem to hold only for the highest luminosity systems (Lutz et al. 2010; Rosario et al. 2012; Rovilos et al. 2012). Other studies suggest little or no connection between the average SFR and black hole accretion (Harrison et al. 2012) or even link luminous AGN activity with a suppression of star formation (Page et al. 2012), in apparent contradiction with torque-limited growth. However, as recently discussed by Hickox et al. (2014), AGN variability may have important consequences for the observed SFR- $\dot{M}_{\mathrm{BH}}$ correlations.

Global changes in star formation occur on timescales comparable to the dynamical timescale of the galaxy while highresolution simulations show that significant AGN variability may occur at essentially all timescales (Hopkins \& Quataert 2010; Levine et al. 2010; Novak et al. 2011; Gabor \& Bournaud 2013). Furthermore, SFR tracers are typically sensitive to star formation events on timescales up to $\sim 100 \mathrm{Myr}$, while the measurements of, for example, X-ray luminosity in AGN track the "instantaneous" black hole accretion rate. Therefore, a direct connection between SFR and AGN activity is only expected when one averages black hole accretion rates over sufficiently long time periods (which is obviously not possible in the observations) or, alternatively, when one calculates the average "instantaneous" accretion rate as a function of host galaxy SFR for a statistical sample of systems. Indeed, this second approach has been pursued recently with results that strongly suggest a coevolution of star formation and black hole accretion on galaxy evolution timescales (Rafferty et al. 2011; Mullaney et al. 2012a; Chen et al. 2013), in agreement with torque-limited growth. Furthermore, Hickox et al. (2014) have shown that accounting for short-term AGN variability may bring a wide range of observations into agreement with an underlying SFR $-\dot{M}_{\mathrm{BH}}$ correlation on cosmological timescales, including the observed weak correlations between SFR and AGN luminosity in normal systems and general trends in the observed AGN luminosity functions. Hence, observations are broadly consistent with the basic prediction of our torque-limited model in that, when averaged over cosmological timescales, black hole accretion rates track their host galaxies' SFRs.

\subsection{Convergence toward the Scaling Relations}

Black holes tend to evolve onto the $M_{\mathrm{BH}}-M_{\text {bulge }}$ relation corresponding to their host galaxy regardless of the initial conditions (Anglés-Alcázar et al. 2013) and with no need for mass averaging through mergers or additional self-regulation processes (Section 3.2). Besides providing a natural explanation for the observed scaling relations, this convergent behavior of 


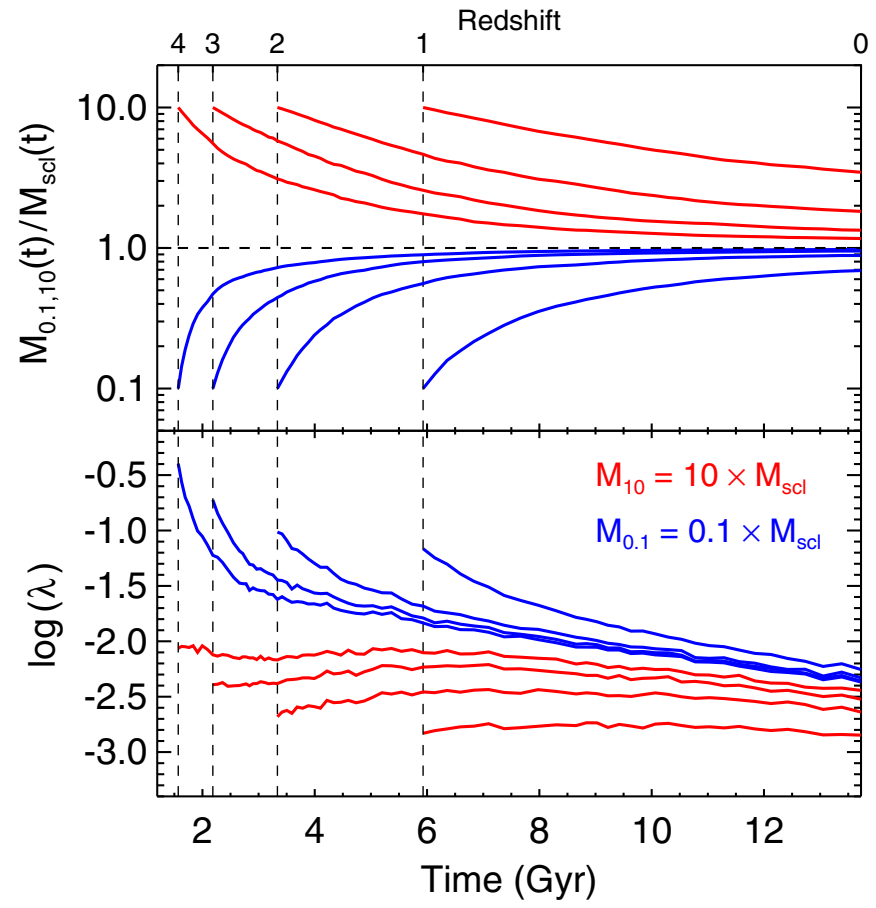

Figure 9. Top: the impact of initial conditions on black hole growth. For each host galaxy, we consider black holes with initial masses that are either a factor of 10 above $\left(M_{10}\right.$; red) or below $\left(M_{0.1}\right.$; blue) the corresponding $M_{\mathrm{BH}}-M_{\text {bulge }}$ relation and compare their evolution to that of a central black hole initially consistent with the $M_{\mathrm{BH}}-M_{\text {bulge }}$ relation $\left(M_{\mathrm{scl}}\right)$. Red and blue solid lines show median values for the mass ratios $M_{10} / M_{\mathrm{scl}}$ and $M_{0.1} / M_{\mathrm{scl}}$, respectively, for all host galaxies as a function of time. Initial conditions are defined at a common redshift for all galaxies, which is taken to be $z=4,3,2$, or 1 , as indicated by the vertical dashed lines. Bottom: evolution of accretion rates in Eddington units resulting from the initial conditions defined in the top panel. Red and blue solid lines correspond to median values for black holes initially over-massive or under-massive relative to the $M_{\mathrm{BH}}-M_{\text {bulge }}$ relation at the starting redshift.

gravitational torque accretion may have significant implications for the accretion histories of massive black holes and the interpretation of observations.

Figure 9 provides further insight into this by comparing the growth of central black holes with different initial masses under the evolution of the same host galaxy. For each of the 213 simulated galaxies from our primary sample, we follow the evolution of three black holes with an initial mass taken to be (1) consistent with the corresponding $M_{\mathrm{BH}}-M_{\text {bulge }}$ relation at $z=4, M_{\mathrm{scl}}$, (2) a factor of 10 above, $M_{10} \equiv 10 \times M_{\mathrm{scl}}$, and (3) a factor of 10 below, $M_{0.1} \equiv 0.1 \times M_{\text {scl }}$. We then calculate the median value of the mass ratios $M_{10}(t) / M_{\mathrm{scl}}(t)$ and $M_{0.1}(t) / M_{\text {scl }}(t)$ over all host galaxies as a function of time, which are shown by the red and blue solid lines in the top panel of Figure 9. The same process is repeated for starting redshifts $z=$ $4,3,2$, and 1 , where all host galaxies are "seeded" at the same redshift using black holes with initial masses as defined above.

As expected from the middle panel of Figure 4, the initial conditions for black hole growth are smoothed out by subsequent evolution, resulting in mass ratios $M_{10}(t) / M_{\mathrm{scl}}(t)$ and $M_{0.1}(t) / M_{\mathrm{scl}}(t)$ that approaches one with time. Figure 9 (top panel) allows us to infer the timescale in which torquelimited growth erases the initial conditions and its dependence on redshift. We find that over-massive black holes require longer convergence timescales relative to black holes with initial mass below the scaling relation. Furthermore, the timescale for convergence toward the $M_{\mathrm{BH}}-M_{\text {bulge }}$ relation significantly increases with decreasing starting redshift. This is seen for initial black holes both above and below the scaling relation.

This numerical experiment allows us to look at the effects of initial conditions on the evolution of Eddington ratios. The bottom panel of Figure 9 shows the evolution of the median Eddington ratios corresponding to the populations of black holes initially over-massive or under-massive at different starting redshifts, as defined for the top panel. Given the dependence of gravitational torque rates on black hole mass $\left(\lambda \propto M_{\mathrm{BH}}^{-5 / 6}\right.$ ), under-massive black holes are characterized by higher Eddington ratios relative to black holes lying on the $M_{\mathrm{BH}}-M_{\text {bulge }}$ relation. Increased Eddington ratios only last for a period of time given by the convergence timescale and, therefore, the evolution of $\lambda$ is characterized by a rapid decrease at early times followed by the usual decline at lower redshifts, as seen in Figure 5. Similar arguments can be made for a population of over-massive black holes at any given redshift. In this case, Eddington ratios are strongly suppressed initially and may even slightly increase with time if the mass decline relative to the scaling relation supersedes the overall decline in Eddington ratios. The net effect of having a population of over-massive black holes relative to the $M_{\mathrm{BH}}-M_{\text {bulge }}$ relation at any given redshift is a weaker evolution of $\lambda$ with time.

Figure 10 shows quantitative predictions of the timescale for convergence toward the $M_{\mathrm{BH}}-M_{\text {bulge }}$ relation, which we define here as the time required for a black hole with initial mass either 10 times above or below to that corresponding to the $M_{\mathrm{BH}}-M_{\text {bulge }}$ relation to grow to less than a factor of two difference relative to a black hole that had an initial mass consistent with the $M_{\mathrm{BH}}-M_{\text {bulge }}$ relation at the starting redshift. We compute black hole convergence probabilities as a function of time after seeding based on the number of host galaxies for which their central black holes did converge in a given timescale. As in Figure 9, we take $z=4,3,2$, and 1 as the starting redshifts. The timescales are expressed in Gyr for the left panel and scaled by the Hubble time corresponding to each starting redshift in the right panel.

The convergence time probability distribution for undermassive black holes peaks at significantly shorter timescales relative to over-massive black holes (Figure 10, left panel). For example, the median convergence timescale for under-massive black holes starting at $z=4$ is $\sim 0.7 \mathrm{Gyr}$ whereas for overmassive black holes it increases up to $\sim 3.6$ Gyr. This is not unexpected, since the amount of mass required to balance out the initial mass difference relative to the baseline mass from the $M_{\mathrm{BH}}-M_{\text {bulge }}$ relation is about 10 times higher for over-massive black holes according to the definition adopted here. Indeed, only $\sim 5 \%$ of the over-massive black holes starting at $z=1$ had enough time to converge before the end of the simulation at $z=$ 0 , while $\sim 86 \%$ of the under-massive black holes starting at $z=$ 1 have converged.

Given some initial log-normal scatter, the mass-dependence of the convergence timescales may produce a bias toward highermass black holes at later times, since it takes longer for highermass black holes to evolve toward the $M_{\mathrm{BH}}-M_{\text {bulge }}$ relation relative to lower-mass black holes. If the intrinsic scatter of the $M_{\mathrm{BH}}-M_{\text {bulge }}$ relation is higher at early times, this might imply an increasing number of over-massive black holes at higher redshifts that could be observed prior to convergence, as some observations suggest (Treu et al. 2007; Decarli et al. 2010; Greene et al. 2010; Merloni et al. 2010; Bennert et al. 2011; Targett et al. 2012). The initial conditions as well as the redshift dependence of the convergence timescales may thus 

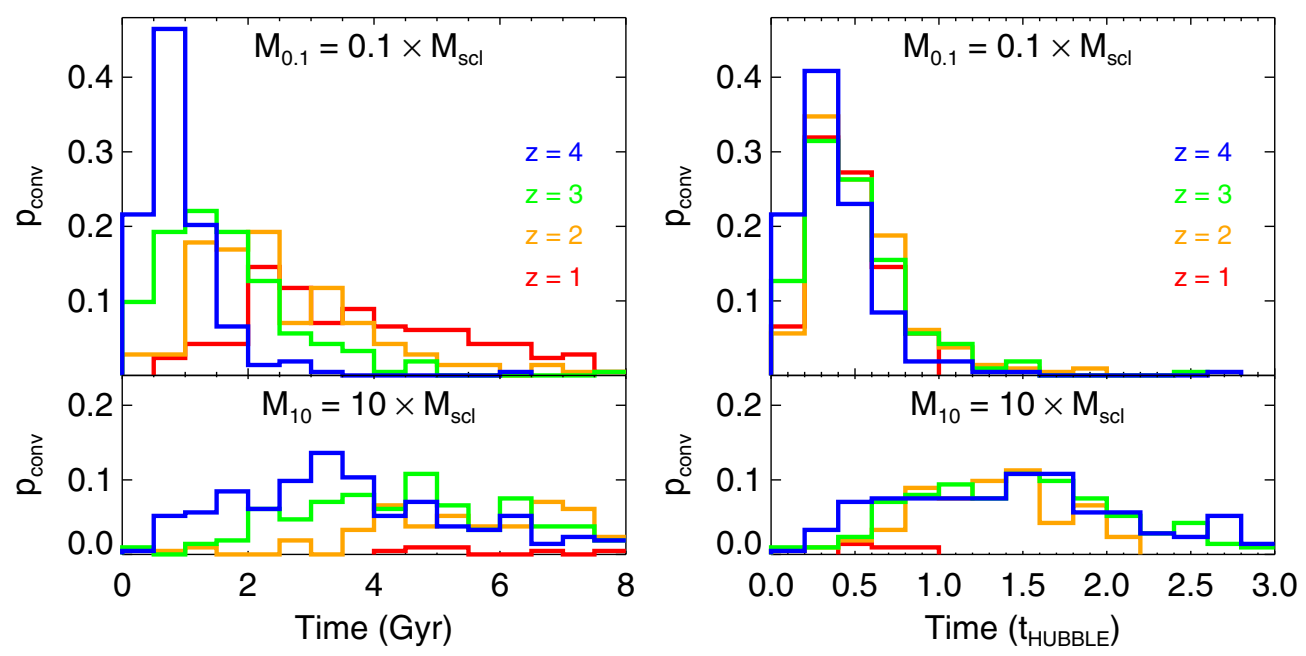

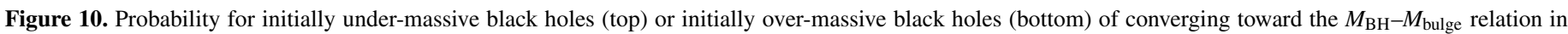

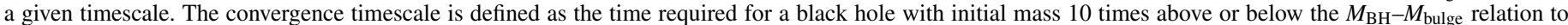

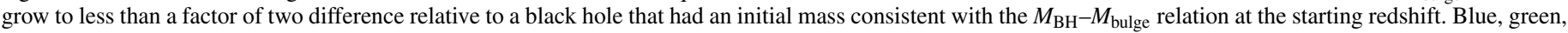

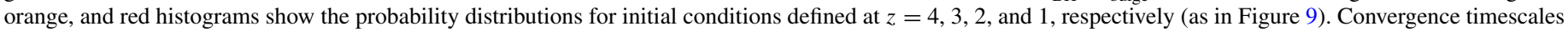
are expressed in units of $1 \mathrm{Gyr}$ (left) or in units of the Hubble time at each redshift (right).

have implications on the observed evolution of black hole mass to host galaxy mass ratios.

Convergence probability distributions are indeed a strong function of starting redshift. The median convergence timescale and the spread of the distribution both increase with decreasing redshift. For under-massive black holes, the characteristic (median) timescale increases from $\sim 0.7$ Gyr for $z=4$ to $\sim 3.5$ Gyr for $z=1$, and the standard deviation of the distribution increases by a factor $\sim 2.6$ with decreasing starting redshift from $z=4 \rightarrow 1$. Similar trends can be seen for the distributions corresponding to over-massive black holes, despite not being appropriately characterized at the lower redshifts given the fraction of black holes for which convergence timescales are not well defined. Interestingly, if we express convergence timescales in units of the Hubble time for each starting redshift, $t_{\text {Hubble }}(z)$, the resulting probability distributions are remarkably similar (Figure 10, right panel). The characteristic timescale for convergence toward the $M_{\mathrm{BH}}-M_{\text {bulge }}$ relation is $\sim 0.5 \times t_{\text {Hubble }}$ and $\sim 1.5 \times t_{\text {Hubble }}$ for under-massive and over-massive black holes, respectively, regardless of the starting redshift. This suggests that cosmological gas infall is the ultimate physical mechanism driving the global evolution of massive black holes and galaxies.

What is making black holes converge toward a similar mass regardless of the initial conditions? Let us consider a generic model in which the accretion rate depends on the black hole mass with some power index $p, \dot{M}_{\mathrm{BH}}=D(t) \times M_{\mathrm{BH}}^{p}$, where $D(t)$ contains all the explicit dependencies on the host galaxy properties. Let us now consider the growth of two seed black holes with masses $M_{\mathrm{a}}(t)$ and $M_{\mathrm{b}}(t)$ evolving at the center of an identical host galaxy. The evolution of their mass ratio is simply given by:

$$
\frac{d}{d t}\left(\frac{M_{\mathrm{a}}}{M_{\mathrm{b}}}\right)=D(t) \frac{M_{\mathrm{a}}^{p}}{M_{\mathrm{b}}}\left[1-\left(\frac{M_{\mathrm{a}}}{M_{\mathrm{b}}}\right)^{1-p}\right],
$$

where we have used $\dot{M}_{\mathrm{a}}=D(t) M_{\mathrm{a}}^{p}, \dot{M}_{\mathrm{b}}=D(t) M_{\mathrm{b}}^{p}$, and the fact the both black holes evolve under the same physical conditions $D(t)$. Therefore, if $p<1$, as is the case for the gravitational torque model (Equation (2)), the mass ratio $m_{\mathrm{ab}} \equiv M_{\mathrm{a}} / M_{\mathrm{b}}$ will tend to approach one regardless of the initial conditions:

$\begin{array}{ll}\text { 1. } d m_{\mathrm{ab}} / d t<0, & \text { if } m_{\mathrm{ab}}>1 \\ \text { 2. } d m_{\mathrm{ab}} / d t>0, & \text { if } m_{\mathrm{ab}}<1 .\end{array}$

Note that the exact opposite result applies to accretion models with $p>1$, including the popular Bondi-Hoyle-Littleton parametrization $(p=2$; Hoyle \& Lyttleton 1939; Bondi \& Hoyle 1944; Bondi 1952) and direct free-fall accretion ( $p=2$; Hobbs et al. 2012). Other examples of accretion parametrizations with $p<1$ include the local viscous accretion rate (Debuhr et al. 2011) and the radiation drag model (Okamoto et al. 2008), neither of which have an explicit dependence on black hole mass $(p=0)$.

The power index $p$ determines whether the initial conditions for black hole growth, i.e., the initial black hole mass, tend to be erased $(p<1)$ or accentuated $(p>1)$ with subsequent evolution. The timescale for which black holes with different masses converge toward a similar value depends on the initial mass ratio and the physical conditions $D(t)$ in the host galaxy. Thus, the spread of the probability distributions in Figure 10 reflect the diversity of accretion histories for our sample of host galaxies. Note, however, that $p<1$ alone does not imply convergence toward the $M_{\mathrm{BH}}-M_{\text {bulge }}$ relation specifically; the slope and normalization is a non-trivial consequence of the physics included in the black hole accretion parametrization.

Equation (8) implies that fine tuning of initial conditions may be required if the main physical mechanism responsible for black hole growth satisfies $p>1$, since slightly different initial conditions could result in rather different black hole masses at later times. Black hole accretion rates are defined to be positive and, therefore, any valid accretion parametrization must satisfy $D(t)>0$ at all times. Thus, the only way to make a black hole model with $p>1$ less sensitive to the initial conditions is by introducing some additional dependence on black hole mass that cannot be absorbed into the power law dependence. In practice, this is accomplished by having $D(t)$ depend on the accretion rate itself, $\dot{M}_{\mathrm{BH}}=D\left(t, \dot{M}_{\mathrm{BH}}\right) \times M_{\mathrm{BH}}^{p}$, which is usually justified in self-regulated models by assuming that feedback from the accretion process has a direct effect on the accretion flow itself. This simple argument shows why a nonlinear feedback loop is 


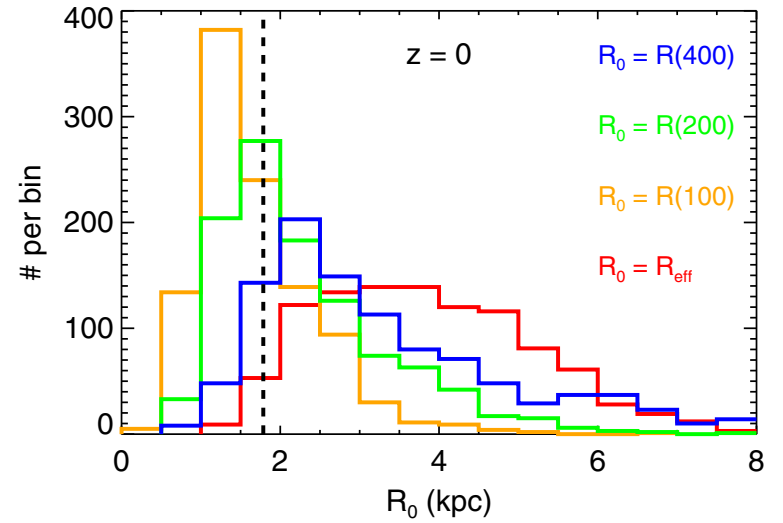

Figure 11. Distribution of radial apertures $\left(R_{0}\right)$ at $z=0$ corresponding to different definitions, including the smallest radius enclosing (1) 100 (orange), (2) 200 (green), or (3) 400 (blue) gas and star particles, and (4) the effective radius of the host galaxy, $R_{\text {eff }}$ (red). All galaxies containing at least 400 gas particles and 400 star particles at $z=0$ have been included. The vertical dashed line indicates the gravitational softening length of the simulation in physical kpc at $z=0$.

required to regulate black hole growth when using an accretion parametrization with a strong dependence on black hole mass (Anglés-Alcázar et al. 2013), and why the torque-limited model does not require explicit self-regulation.

\section{NUMERICAL ROBUSTNESS}

In Anglés-Alcázar et al. (2013), we conducted an extensive analysis of how the inferred gravitational torque rates and the resulting black hole-galaxy scaling relations depend on a variety of parameters and implementation details including: numerical resolution, the mass retention rate $\left(\epsilon_{\mathrm{m}}\right)$, and different stellar feedback models. In this section, we complement our previous study by presenting additional tests, focusing primarily on the convergence of results for different radial apertures $\left(R_{0}\right)$ and on the impact of our quenching prescription on the co-evolution of black holes and galaxies. Specific numerical convergence tests for our bulge-disk decomposition procedure are presented in the Appendix, where a direct comparison is made to other decomposition methods commonly used in the literature.

\subsection{Evaluating Gravitational Torque Rates}

We select all galaxies containing at least 400 gas particles and 400 star particles at $z=0$, so that different radial apertures can be defined for a common galaxy sample. To evaluate the effects of $R_{0}$ on the inferred gravitational torque rates, we define radial apertures that enclose at least either (1) $100\left(R_{100}\right)$, (2) 200 $\left(R_{200}\right)$, or (3) $400\left(R_{400}\right)$ gas and star particles, or, alternatively, we use (4) the effective radius of the host galaxy, $R_{0}=R_{\text {eff }}$, as in Section 3.5. Figure 11 shows the distribution of radial apertures at $z=0$ corresponding to the different definitions.

Figure 12 shows some of the key galaxy properties entering into the calculation of gravitational torque rates as a function of black hole mass at $z=0$. In particular, we calculate the disk fraction $\left(f_{\mathrm{d}}\right)$, the gas fraction relative to the disk mass $\left(f_{\text {gas }}\right)$, and the total disk mass within $R_{0}\left(M_{\mathrm{d}}\right)$ for the radial apertures defined above. In addition, we evaluate the accretion rates as well as the Eddington ratios corresponding to the different radial apertures by assuming that black holes lie on the $M_{\mathrm{BH}}-M_{\text {bulge }}$ relation with 0.5 dex scatter in black hole mass.

The top panel of Figure 12 shows that there is significant scatter in the disk fraction, with median $f_{\mathrm{d}}$ values roughly

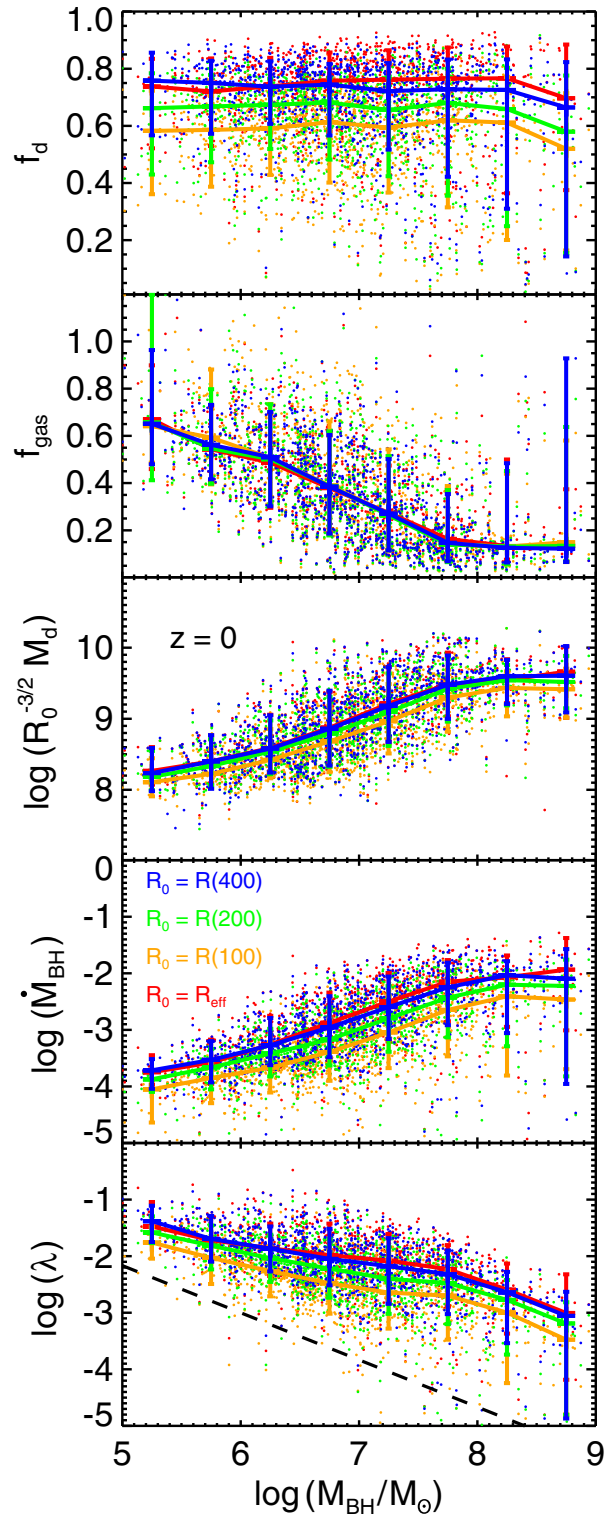

Figure 12. Effects of different radial apertures $\left(R_{0}\right)$ on the inferred gravitational torque rates for all galaxies with at least 400 gas and star particles at $z=0$. Accretion rates are calculated for different definitions of the radial aperture $R_{0}$, including the smallest radius enclosing (1) 100 (orange), (2) 200 (green), or (3) 400 (blue) gas and star particles, and (4) the effective radius of the host galaxy, $R_{\text {eff }}$ (red). We show, from top to bottom, some of the key physical quantities defined in Equation (2) as a function of black hole mass: $f_{\mathrm{d}}, f_{\text {gas }}$, and $R_{0}^{-3 / 2} M_{\mathrm{d}}$ (in units of $\mathrm{kpc}^{-3 / 2} M_{\odot}$ ), as well as the resulting accretion rates (in units of $M_{\odot} \mathrm{yr}^{-1}$ ) and Eddington ratios, where we have assumed that black holes lie on the $M_{\mathrm{BH}}-M_{\text {bulge }}$ relation with 0.5 dex scatter in black hole mass. Individual black holes are represented by points with the colors corresponding to the different radial apertures. Points with error bars connected by solid lines show median values within logarithmically spaced bins in $M_{\mathrm{BH}}$ and the central $75 \%$ of the distribution. The black dashed line in the bottom panel corresponds to the scaling $\lambda \propto M_{\mathrm{BH}}^{-5 / 6}$.

independent of black hole mass independent of the definition of $R_{0}$. There is actually an increasing number of black holes with host galaxies having lower $f_{\mathrm{d}}$ values at higher masses, but this trend is not as clear as we would expect from observations in the local universe, where the majority of $\sim 10^{9} M_{\odot}$ black holes reside in elliptical galaxies. This is, however, not surprising given the low number of the most massive systems in our simulation as well as the definition of disk fraction employed 
here, which may include contributions from rotating bulges (Section 2.4). Nonetheless, we find evidence for a systematic trend of increasing disk fractions for larger radial apertures. Median $f_{\mathrm{d}}$ values within logarithmically spaced bins in $M_{\mathrm{BH}}$, indicated by points with error bars connected by solid lines, increase by about $25 \%$ for the radial aperture $R_{100}$ relative to $R_{400}$. This might be expected given that, at the resolution of our cosmological simulation, $R_{400}$ is comparable to the effective radius $\left(R_{\text {eff }}\right)$ and galaxies are more likely bulge-dominated in their central regions. Furthermore, the radial apertures $R_{100}$ and $R_{200}$ are comparable to or even smaller than the gravitational softening length of the simulation (Figure 11). The lack of gravitational resolution could result in the overestimation of bulge masses (and hence the underestimation of $f_{\mathrm{d}}$ ) owing to the increased random motions and the overall reduction in the amount of ordered circular motion for an artificially shallower gravitational potential (Anglés-Alcázar et al. 2014). Note, however, that this is greatly compensated by our definition of $f_{\mathrm{d}}$ (formally an upper limit to the disk fraction), resulting in better resolution convergence relative to more restrictive bulge-disk decomposition methods (the Appendix).

Gas fractions relative to the disk mass are better converged with respect to $R_{0}$, as shown in the second panel of Figure 12 (from top to bottom). The median values of $f_{\text {gas }}$ are essentially coincident for the different radial apertures, clearly decreasing for higher mass black holes and, therefore, higher mass galaxies, as expected (Davé et al. 2011a). Note that $f_{\text {gas }}$ here is defined as the ratio of the gas mass to the total disk mass and may thus be greater than one (Equation (4)).

The disk mass normalized by the radial aperture, $R_{0}^{-3 / 2} M_{\mathrm{d}}$, shows, again, some discrepancy between the different definitions of $R_{0}$. The radial aperture $R_{400}$ yields median values about 0.2 dex above those obtained with $R_{100}$. Besides the obvious dependence on $f_{\mathrm{d}}$, different radial density profiles may also affect the inferred $R_{0}^{-3 / 2} M_{\mathrm{d}}$ values. Indeed, the comparison between $R_{400}$ and $R_{200}$ suggests that we are approaching numerical convergence. Interestingly, all radial apertures show a significant increase in median $R_{0}^{-3 / 2} M_{\mathrm{d}}$ values with increasing black hole mass. Given that there is no clear trend for $f_{\mathrm{d}}$ with $M_{\mathrm{BH}}$, this suggests that more massive galaxies are more compact.

The bottom two panels of Figure 12 show the accretion rates and Eddington ratios as a function of $M_{\mathrm{BH}}$ resulting from the galaxy properties described in the upper panels. The combined effects of the radial aperture result in median $\dot{M}_{\mathrm{BH}}$ and $\lambda$ values that may vary by about a factor of two to three for the different definitions of $R_{0}$. Despite this, all radial apertures considered here yield very similar trends with $M_{\mathrm{BH}}$ and, therefore, a simple normalization factor may bring the results into better agreement. Note that the radial aperture must be chosen as a trade off between (1) the number of resolution elements required to characterize the morphology of the galaxy and (2) smaller physical radial apertures that provide a better prediction for the gas inflows at sub-parsec scales (Hopkins \& Quataert 2011). Thus, strict convergence with respect to $R_{0}$ may only be reached by increasing the mass and force resolution of the simulation (Anglés-Alcázar et al. 2013).

Besides illustrating the robustness of our methodology to changes in $R_{0}$ at fixed resolution, Figure 12 allows us to gain further insight into the dependence of Eddington ratios on black hole mass. Indeed, the increased dynamic range relative to our baseline sample of 213 black holes (Figure 6) reveals a clear trend for $\lambda$ values to decrease with $M_{\mathrm{BH}}$. Similar results are reproduced at all redshifts, with consistently weaker trends relative to the expected $\lambda \propto M_{\mathrm{BH}}^{-5 / 6}$ dependence, as described in Section 3.3. Higher mass galaxies are more compact than lower mass galaxies while having a similar disk fraction within $R_{0}$, resulting in higher values of $R_{0}^{-3 / 2} M_{\mathrm{d}}$. Moreover, Figure 12 shows that, at fixed redshift, accretion rates are primarily determined by $R_{0}^{-3 / 2} M_{\mathrm{d}}$, yielding higher $\dot{M}_{\mathrm{BH}}$ values with increasing $M_{\mathrm{BH}}$. Note that the gas fraction decreases with increasing $M_{\mathrm{BH}}$ but the torque model is only weakly dependent on $f_{\text {gas. }}$. The resulting accretion rates, when normalized by black hole mass, yield Eddington ratios decreasing with $M_{\mathrm{BH}}$ roughly as $\lambda \propto M_{\mathrm{BH}}^{-1 / 2}$. We do not pursue this further given the limited mass range of the sample of our 213 black hole sample for which their evolution can be constrained self-consistently from $z \gtrsim 4$ down to $z=0$.

\subsection{The $S F R-\dot{M}_{B H}$ Relation}

We now turn back to the SFR- $\dot{M}_{\mathrm{BH}}$ diagram in Figure 13, where we evaluate the effects of different radial apertures and numerical resolution on the inferred SFR-AGN connection at $z=2$. As in Figure 12, we calculate accretion rates using different values of $R_{0}$ and assuming that black holes lie on the $M_{\mathrm{BH}}-M_{\text {bulge }}$ relation with 0.5 dex scatter in black hole mass. However, we include here all $z=2$ host galaxies with at least the minimum number of particles sufficient to define each of the different radial apertures; this results in a significantly larger dynamic range for $R_{0}=R_{\text {eff }}$ relative to, for example, $R_{0}=R_{200}$ since the effective radius can be defined for all galaxies.

The left panel of Figure 13 shows the median SFRs within logarithmically spaced bins in $\dot{M}_{\mathrm{BH}}$ corresponding to the different radial apertures, with error bars indicating the 12.5 and 87.5 percentiles. Besides the increased dynamic range, smaller apertures yield higher SFRs for a given accretion rate, corresponding to higher mass galaxies and higher mass black holes, as expected from our discussion in Section 4.1. However, this is not the case in the low $\dot{M}_{\mathrm{BH}}$ regime, where the SFR- $\dot{M}_{\mathrm{BH}}$ relation flattens out and smaller radial apertures seem to result in lower SFRs for a given $\dot{M}_{\mathrm{BH}}$. This partly owes to selection effects, since larger radial apertures can be defined only for higher mass galaxies with correspondingly higher SFRs.

At a more fundamental level, one expects a flattening of the SFR $-\dot{M}_{\mathrm{BH}}$ relation at low $\dot{M}_{\mathrm{BH}}$ to occur when calculating median SFRs as a function of $\dot{M}_{\mathrm{BH}}$ owing to the different characteristic variability timescales of star formation and black hole accretion and because black holes spend more time accreting below the mean value (Hickox et al. 2014). This trend disappears when we invert the $\mathrm{SFR}-M_{\mathrm{BH}}$ relation and calculate median black hole accretion rates within SFR bins, as shown in the middle panel of Figure 13. In this case, the gravitational torque model produces an SFR $-\dot{M}_{\mathrm{BH}}$ relation similar to that of Chen et al. (2013) even in the low $\dot{M}_{\mathrm{BH}}$ regime and for all radial apertures. Note that the scatter increases for higher $\dot{M}_{\mathrm{BH}}$ values owing to the small number of galaxies with high SFRs.

As an additional consistency check, the right panel of Figure 13 shows the location in the SFR- $\dot{M}_{\mathrm{BH}}$ plane of eight $z=2$ re-simulated galaxies taken form Anglés-Alcázar et al. (2013). The higher resolution of these cosmological zoom simulations (a 20 times higher mass resolution relative to our primary cosmological simulation in this work) allowed us to compute galaxy properties within a fixed radial aperture $R_{0}=1 \mathrm{kpc}$ (physical) for all galaxies at all times. Encouragingly, the 

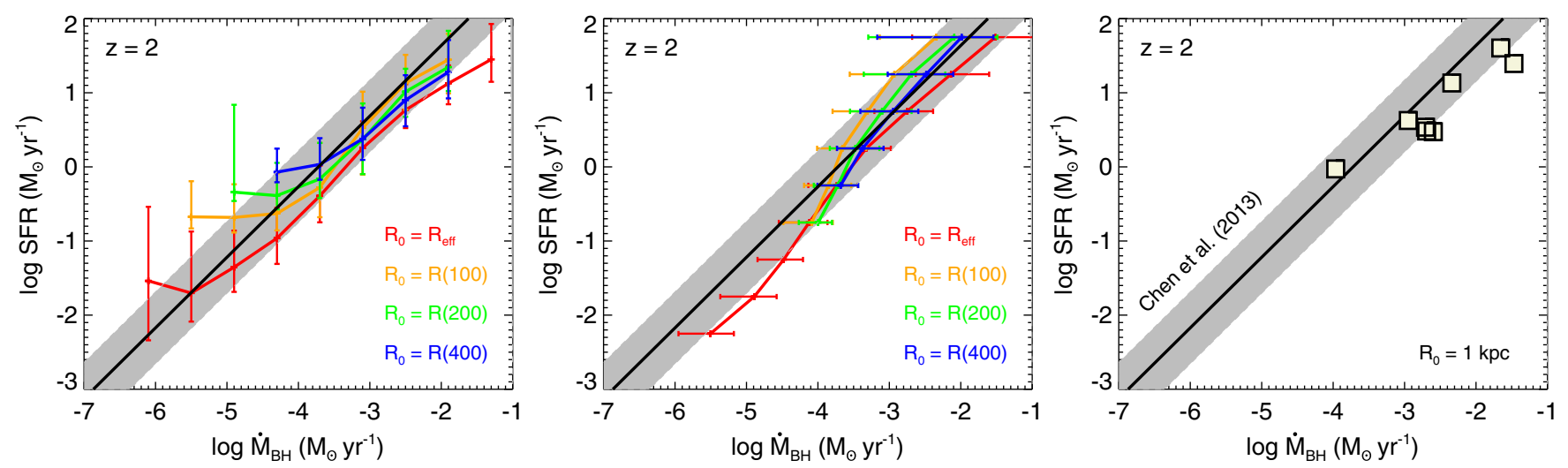

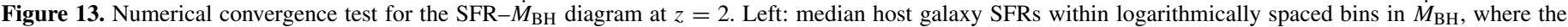

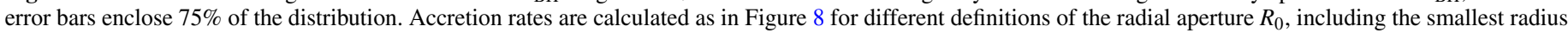

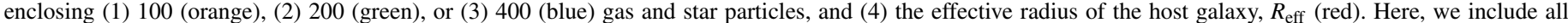

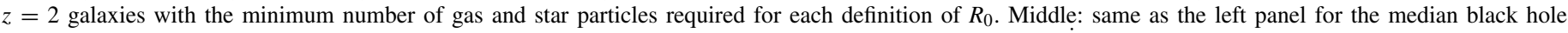

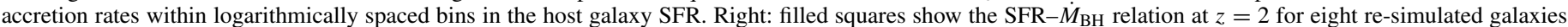

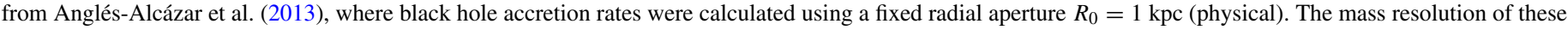

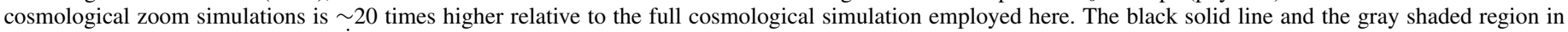
each panel correspond to the SFR $-\dot{M}_{\mathrm{BH}}$ relation of Chen et al. (2013) and the estimated uncertainty in the normalization.

inferred central black holes occupy the region of the diagram expected for their host galaxy SFRs, in agreement with our current results and independent of the differences in resolution, the operational definition of $R_{0}$, and even feedback effects, since these simulations did not include any quenching mechanism.

\subsection{Effects of Star-formation Quenching}

Our primary cosmological simulation incorporates a heuristic prescription for star-formation quenching that is tuned to reproduce the observed exponential cutoff in the high-mass end of the stellar mass function at $z=0$ (Davé et al. 2013). Given that AGN feedback is currently the best candidate for suppressing star formation in the high-mass regime, it is important to evaluate the impact of our quenching prescription on the inferred co-evolution of black holes and galaxies. To do so, we have repeated all calculations for an additional cosmological simulation with the same size and resolution but including no quenching mechanism. Specifically, this is the r32n512vzw run described in Davé et al. (2013). We note that this simulation adopts a slightly different model for galactic outflows, but the differences are confined to galaxies with velocity dispersions $\sigma<75 \mathrm{~km} \mathrm{~s}^{-1}$, whereas the galaxies we consider here are generally larger. Hence the main difference for black hole growth should reflect the differences owing to our heuristic quenching prescription.

Figure 14 shows the $M_{\mathrm{BH}}-M_{\text {bulge }}$ relation obtained at different redshifts when we include no quenching prescription. Here, we have applied the same selection criteria as in Section 3.2, including only galaxies that contain at least 200 gas and 200 star particles at all times and that are identified in the cosmological simulation as early as $z \geqslant 4$. This results in a higher number of galaxies extending to higher masses relative to the simulation with star-formation quenching. Despite the strong difference at the high-mass end of the stellar mass function, our no-quenching simulation yields black holes and host galaxies evolving on average along the $M_{\mathrm{BH}}-M_{\text {bulge }}$ relation, just as in our quenching simulation (see the bottom panel of Figure 4), and for the same mass retention rate $\epsilon_{\mathrm{m}}=0.05$. Interpreting our heuristic quenching prescription as a plausible effect of AGN feedback acting on the host galaxy, this would suggest that AGN feedback is not driving the connection between black holes and galaxies even if it is responsible for star-formation quenching. All other

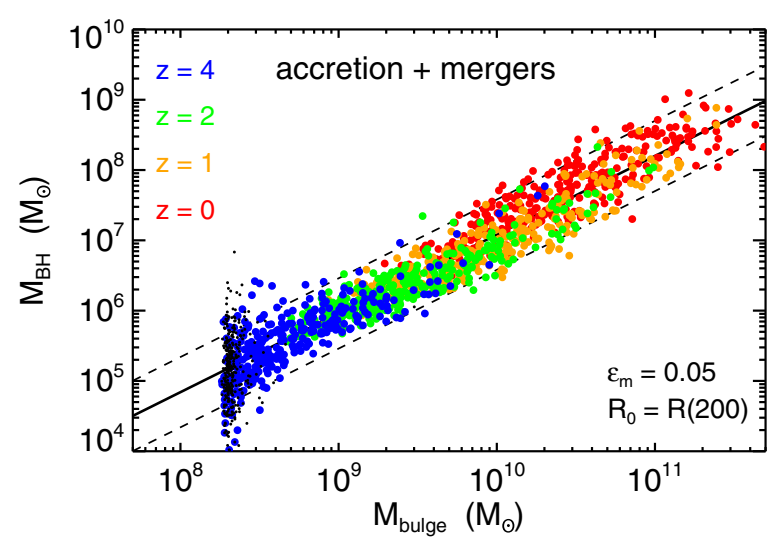

Figure 14. $M_{\mathrm{BH}}-M_{\text {bulge }}$ relation at $z=0$ (red), 1 (orange), 2 (green), and 4 (blue) for black holes growing through torque-limited accretion and mergers. Host galaxies are taken from a $2 \times 512^{3}$ cosmological simulation in a $\left[32 h^{-1} \mathrm{Mpc}\right]^{3}$ comoving box including no high mass galaxy quenching mechanism. Masses of black hole seeds (shown as small black dots) and merging black holes are randomly selected from a log-normal distribution corresponding to the $M_{\mathrm{BH}}-M_{\text {bulge }}$ relation for the appropriate galaxy and time step, assuming a $0.5 \mathrm{dex}$ scatter in black hole mass. The black solid line shows the $M_{\mathrm{BH}}-M_{\text {bulge }}$ relation of Häring \& Rix (2004) while the black dashed lines indicate a 0.5 dex scatter in black hole mass.

properties of torque-limited growth analyzed here such as the convergence toward the scaling relations and the characteristic distribution and evolution of Eddington ratios are reproduced by our no-quenching simulation, providing further support for the numerical robustness of our methodology.

Overall, our results are mildly sensitive to the radial aperture considered, in the sense that smaller apertures result in lower accretion rates. However, the overall trends are unchanged, hence a modest re-normalization of $\epsilon_{m}$ could compensate for the differences, and our general conclusions are unaffected by this choice. Even though our heuristic quenching prescription results in significantly fewer massive galaxies, it has little impact on the black hole properties at a given bulge mass.

\section{DISCUSSION AND CONCLUSIONS}

In this paper, we applied and extended the methodology developed in Anglés-Alcázar et al. (2013) to infer the role of accretion 
driven by gravitational torques on the evolution of massive black holes at the centers of star-forming galaxies over cosmic time. By combining the analytic model of Hopkins \& Quataert (2011) with full cosmological hydrodynamics simulations, we have (1) constrained the physics driving the observed black hole-galaxy scaling relations, (2) evaluated the relative importance of black hole mergers for the total growth, (3) presented predictions for the distribution and evolution of Eddington ratios, and (4) investigated the global connection between black hole accretion and star formation. Our main results can be summarized as follows.

1. Torque-limited growth yields black holes and host galaxies evolving on average along the $M_{\mathrm{BH}}-M_{\text {bulge }}$ relation from early times down to $z=0$. The normalization of the scaling relation depends on the mass retention rate $\epsilon_{\mathrm{m}}$, which represents the fraction of the inflowing gas feeding the accretion disk from galactic scales that is finally accreted by the central black hole. We find that $\epsilon_{\mathrm{m}} \approx 5 \%$ provides an appropriate normalization, implying that $\sim 95 \%$ of the mass inflow at sub-parsec scales does not make it onto the black hole and may be lost to winds and outflows.

2. By identifying galaxy mergers down to mass ratios of 1:5, we find that smooth accretion represents most of black hole growth and dominates the overall evolution in the $M_{\mathrm{BH}}-M_{\text {bulge }}$ plane. Black hole mergers represent typically a small fraction of the total growth except in some exceptional cases with numerous mergers happening preferentially at low redshift.

3. Black holes tend to evolve onto the $M_{\mathrm{BH}}-M_{\text {bulge }}$ relation corresponding to their host galaxy independent of the initial conditions and with no need for mass averaging through mergers or additional self-regulation processes. The characteristic convergence timescale for black holes starting a factor of 10 above or below the $M_{\mathrm{BH}}-M_{\text {bulge }}$ relation is about 0.5 and 1.5 times the Hubble time for initially undermassive and over-massive black holes, respectively.

4. The weak dependence of gravitational torque rates on black hole mass plays a key role in the overall convergence behavior. For accretion parametrizations of type $\dot{M}_{\mathrm{BH}} \propto$ $M_{\mathrm{BH}}^{p}$, it can be shown that the power index $p$ determines whether the initial conditions for black hole growth tend to be erased $(p<1)$, as is the case for the gravitational torque model $(p=1 / 6)$, or accentuated $(p>1)$ with subsequent evolution. This implies the need for additional feedback self-regulation for accretion models strongly dependent on $M_{\mathrm{BH}}$, such as the popular Bondi parametrization $(p=2)$.

5. Eddington ratios averaged over galaxy evolution timescales can be described at all redshifts by a broad log-normal distribution with a median value evolving roughly as $\lambda_{\mathrm{MS}} \propto(1+z)^{1.9}$, suggesting the existence of a main sequence for AGN analogous to the cosmic evolution of specific SFRs. Torque-limited accretion yields typical average Eddington ratios $\lambda>10 \%$ at early times, a smooth average transition between radiatively efficient to radiatively inefficient accretion modes at $z \approx 1-2$, and typical present day values of $\lambda \lesssim 1 \%$.

6. The width of the distribution of Eddington ratios increases at lower redshifts, with an extended tail toward low luminosities in radiatively inefficient accretion. The combined dependence of gravitational torque rates on black hole mass and gas+stellar surface density yields a weak trend of decreasing median Eddington ratios with increasing black hole mass at all redshifts.
7. Torque-limited growth predicts a connection between SFR and AGN activity on timescales comparable to the dynamical timescale of galaxies, resulting in a close-to-linear $\left\langle\dot{M}_{\mathrm{BH}}\right\rangle \propto$ SFR relation for the average black hole accretion rate. Cosmological gas infall and transport of angular momentum in the galaxy by gravitational instabilities appear to be the primary drivers of this relation. The SFR-AGN connection can have large fluctuations on a galaxy-by-galaxy basis given the strong variability of black hole accretion at all timescales.

Encouragingly, our main results are robust to changes in a variety of implementation details. Despite the apparently complicated form of the gravitational torque model, by comparing to our previous zoom runs, its application to simulations of different resolution yields reasonably good numerical convergence. We have identified a slight trend for higher accretion rates with increasing radial aperture $R_{0}$ at fixed resolution, suggesting that higher resolution simulations are needed when $R_{0}$ becomes comparable to the effective radius of the galaxy. Nonetheless, the uncertainty in $R_{0}$ described here seems reasonable compared to the typical uncertainties of self-regulated accretion models on, e.g., the frequency at which feedback events occur or the number of resolution elements over which feedback energy or momentum is injected (e.g., Booth \& Schaye 2009; Dubois et al. 2012; Choi et al. 2012; Newton \& Kay 2013). At a sub-grid level, it is assumed that cooling is efficient and most of the gas forms a rotationally supported disk, but the gravitational torque model does not explicitly depend on the resolved gas thermodynamics (Hopkins \& Quataert 2011), which is a major source of uncertainty in galaxy formation simulations. In addition, torque-limited growth yields roughly similar results regardless of stellar feedback effects (Anglés-Alcázar et al. 2013). Different parametrizations of star formation (via an effective equation of state) or stellar feedback may yield rather different structural properties of galaxies (Anglés-Alcázar et al. 2014), but the scalings arising for the response of gas+stellar systems to gravitational torques remain the same: black holes and galaxies simply move along the scaling relations without changing the overall behavior of the gravitational torque model.

Our combined results imply a fuelling-controlled scenario in which black hole growth is primarily governed by the amount of gas supply from galactic scales and not by the direct interaction of feedback energy or momentum from the accretion flow with the surrounding galaxy ISM (Escala 2006, 2007). AGN feedback in the form of winds and outflows from the accretion flow may have a strong impact on the host galaxy and actually represents a significant mass loss relative to the available gas supply, thereby strongly suppressing black hole growth. However, it is the rate at which non-axisymmetric perturbations to the stellar potential drive gas into shocks that dissipate energy and angular momentum that determines the overall rate of black hole growth and the connection between star formation and AGN activity on cosmological timescales.

There remain, however, significant uncertainties that require further investigation. A key assumption in this work is that only a small fraction of the gas feeding the accretion disk from galactic scales is finally accreted by the black hole. We have parametrized the mass lost to winds and outflows in the accretion disk with the simplest possible model, by assuming a constant average mass retention rate $\left(\epsilon_{\mathrm{m}}\right)$ for all black holes at all times. Its value, $\epsilon_{\mathrm{m}}=0.05$, has been determined by requiring consistency with the $M_{\mathrm{BH}}-M_{\text {bulge }}$ relation of Häring \& Rix (2004), but it is subject to uncertainties including a degeneracy with the nuclear star 
formation law or the exact definition of the $M_{\mathrm{BH}}-M_{\text {bulge }}$ relation (Hopkins \& Quataert 2011; Anglés-Alcázar et al. 2013). Despite this simplistic assumption, the inferred average mass retention rate seems roughly consistent with a number of observational studies and theoretical expectations.

Outflows appear to be a common feature of geometrically thick accretion disks, usually ascribed to radiatively inefficient flows forming at very low Eddington ratios $(\lambda \lesssim 0.01$; Narayan $\&$ McClintock 2008), or "slim disks" forming at super-critical rates $(\lambda \gtrsim 1$; Abramowicz et al. 1988). A wide range of simulations of hot accretion flows show strong outflows that may carry away a significant fraction of the mass inflow rate (Yuan et al. 2012; Bu et al. 2013; Li et al. 2013; Sadowski et al. 2013). The radial profile of the mass outflow rate can be described as a power-law in radius, $\dot{M}_{\text {wind }}(r) \propto r^{s}$, with typical power index values $s \sim 0.4-1$ (Yuan et al. 2012). Thus, the black hole accretion rate can be calculated in terms of the torque-limited inflow rate as

$$
\dot{M}_{\mathrm{BH}}=\left[1+\left(\frac{r_{\text {out }}}{r_{\text {in }}}\right)^{s}\right]^{-1} \dot{M}_{\text {Torque }},
$$

where $r_{\text {in }}$ and $r_{\text {out }}$ represent the inner and outer radii of the region where accretion-driven outflows are launched and $\dot{M}_{\text {Torque }}$ represents the gas inflow rate at $r_{\text {out }}$ driven by gravitational instabilities at larger scales. If we take $s=0.5$ (e.g., Bu et al. 2013) and assume that the radial extent of the accretion disk powering outflows spans between two and four orders of magnitude relative to the inner radius, $r_{\text {out }} / r_{\text {in }} \approx 10^{2}-10^{4}$ (Tombesi et al. 2012), we obtain a range for the effective mass retention rate $\epsilon_{\mathrm{m}} \approx 1-10 \%$, roughly consistent with our inferred value. It is less clear from simulations whether radiatively efficient, geometrically thin disks (Shakura \& Sunyaev 1973) produce outflows with similar scalings (Li et al. 2013; Sadowski et al. 2013), but significant mass loss through magnetically or radiationdriven winds may also occur (Proga et al. 2008; Ohsuga \& Mineshige 2011); nonetheless, there is substantial observational evidence for strong outflows in the "quasar mode" feedback, corresponding to radiatively efficient accretion (Fabian 2012).

It is illustrative to obtain a rough estimate of the average kinetic power implied in our model. The kinetic luminosity can be defined in terms of the kinetic efficiency $\epsilon_{\mathrm{k}}$ :

$$
\begin{aligned}
L_{\mathrm{k}} & =\frac{1}{2} \dot{M}_{\text {wind }} v^{2} \equiv \epsilon_{\mathrm{k}} \dot{M}_{\mathrm{BH}} c^{2}, \\
\epsilon_{\mathrm{k}} & =\frac{1}{2}\left(\frac{v}{c}\right)^{2}\left(\frac{1-\epsilon_{\mathrm{m}}}{\epsilon_{\mathrm{m}}}\right),
\end{aligned}
$$

where $v$ is the outflow velocity and the definition of $\epsilon_{\mathrm{m}}$ implies $\dot{M}_{\mathrm{BH}}=\dot{M}_{\text {Torque }}-\dot{M}_{\text {wind }}$. Thus, for mass-weighted outflow velocities in the range $10^{3}-10^{4} \mathrm{~km} \mathrm{~s}^{-1}$, we obtain a plausible range for the kinetic efficiency $\epsilon_{\mathrm{k}} \approx 10^{-4}-10^{-2}$, or between $0.1 \%-10 \%$ of the bolometric luminosity assuming $\eta=0.1$. For comparison, the AGN synthesis model of Merloni \& Heinz (2008) yields a total integrated average kinetic efficiency $\epsilon_{\mathrm{k}} \approx$ $3-5 \times 10^{-3}$, and self-regulated models of black hole growth in galaxy-scale simulations require $\sim 0.5 \%-15 \%$ of the bolometric luminosity to be injected into the surrounding medium in order to affect the host galaxy and possibly reproduce the observed black hole-galaxy scaling relations (Di Matteo et al. 2005; Hopkins \& Elvis 2010; Dubois et al. 2012).

Observations show that winds and outflows are ubiquitous in AGN for a wide range of host galaxy properties (Reynolds 1997;
Veilleux et al. 2005; Fabian 2012), though direct constraints on mass outflow rates have proven difficult to obtain. Tombesi et al. (2012) find mass loss rates $\dot{M}_{\text {wind }} / \dot{M}_{\mathrm{BH}} \sim 0.05$ for ultrafast outflows in radio-quiet AGNs, significantly lower than the mass loss required by torque-limited growth, while a comparison between kinetic wind luminosity to bolometric luminosity for the sample of AGN analyzed by King et al. (2013) suggests typical ratios of mass loss in winds to black hole accretion comparable to our expectations. The inferred outflowing masses and velocities likely depend on the distance from the black hole to the wind material (Tombesi et al. 2013) and the amount of entrainment of surrounding material. Indeed, the total mass-loss in galaxy-scale AGN-driven outflows may exceed the SFR of the entire galaxy (Feruglio et al. 2010; Rupke \& Veilleux 2011; Sturm et al. 2011; Maiolino et al. 2012).

At present, it is difficult to assess how much mass is lost in outflows relative to the gas inflowing at a given radius, but future observations and numerical simulations will provide tighter constraints. Despite the complexity inherent to black hole accretion flows and outflows, it is encouraging that the average mass retention rate required by torque-limited growth on galaxy evolution time scales lies within the range of plausible values. More detailed modeling could include an explicit dependence of the mass retention rate on the accretion mode and should account for the effects of AGN feedback on the host galaxy.

We have shown that there is no need for a strong redshift dependence of the mass retention rate to regulate black hole growth, at least for the range of black hole masses considered here. This suggests that, to first order, the net effect of winds and outflows from the accretion flow is to suppress the instantaneous accretion rate by an average constant factor, contributing significantly to the normalization of the AGN main sequence but only to second order in the slope. The rate of gas supply from galactic scales by gravitational torques dominates the overall evolution of black hole growth on cosmological timescales, sets the slope of the AGN main sequence, and yields the average connection between SFR and AGN activity in star forming galaxies.

Assessing the full validity of our results will require explicit modeling of the interaction between accretion-driven outflows and the surrounding gas, which will be the subject of future work. We speculate that AGN-driven outflows as required by torque-limited growth may have a limited impact on the host galaxy even if significant entrainment of cold ISM gas occurs. Jet heating of hot halo gas may however have a progressive longterm cumulative impact not only on the host galaxy but on black hole growth itself, linked to the overall decrease of cosmological gas infall at lower redshifts and the increasing frequency of radiatively inefficient accretion. Cosmological simulations have shown that preventing gas accretion in hot halos may yield a galaxy red sequence and luminosity function as observed (Gabor \& Davé 2012). Observations of powerful jets generated by a central black hole accreting at low Eddington ratios imply heating rates that are comparable to the cooling rates of hot gas in halos (McNamara \& Nulsen 2007; Fabian 2012). Radio-mode feedback may thus prevent gas accretion into galaxies, reduce their overall star formation, and possibly limit the amount of gas available for black hole accretion (Okamoto et al. 2008).

The late time evolution of massive black holes in quiescent galaxies could, therefore, be self-regulated by a "true" largescale feedback loop, where the gravitational torque model may no longer be an appropriate accretion parametrization. Such a feedback loop in radio mode is, however, unlikely to account for the majority of black hole growth, expected 
to occur through radiatively efficient accretion (Soltan 1982) in gas-rich star-forming galaxies (Heckman \& Best 2014). A hint for a switch in fuelling mechanism from torque-limited growth to self-regulation in radio-mode may be given by observations of Eddington ratio distributions at low redshift, with a characteristic transition from log-normal to power-law distributions in star forming galaxies and passive galaxies, respectively (Kauffmann \& Heckman 2009). We will explicitly address the impact of AGN feedback on the co-evolution of black holes and galaxies in future work, by performing selfconsistent simulations of torque-limited growth and AGNdriven outflows in a cosmological context.

We thank A. Escala, X. Fan, P. Hopkins, and B. Robertson for stimulating conversations. We thank the anonymous referee for a thoughtful report that helped improve the paper. F.Ö. gratefully acknowledges support from the Radcliffe Institute for Advanced Study at Harvard University. R.D. acknowledges support from the South African Research Chair Initiative. The simulations were run on the University of Arizona's 512-processor SGI Altix system and the TACC Sun Constellation Cluster (Ranger) at The University of Texas, Austin. This work used the Extreme Science and Engineering Discovery Environment (XSEDE), which is supported by National Science Foundation grant number OCI-1053575. This work was supported by the National Science Foundation under grant numbers AST-0907998 and AST-1108753 and through a grant from the Ahmanson Foundation. This work was supported in part by the National Science Foundation under grant No. PHYS-1066293 and the hospitality of the Aspen Center for Physics. This work was also supported by NASA Astrophysics Theory grants NNX12AH86G and NNX10AJ95G.

\section{APPENDIX}

\section{CONVERGENCE OF BULGE-DISK DECOMPOSITION}

Different bulge-disk decomposition procedures are possible for the evaluation of black hole accretion rates through Equations (1) and (2) (Section 2.4). Here, we evaluate the numerical convergence properties of three such methods to show that the simple kinematic decomposition used in this work appears to be more robust at the resolution of our cosmological simulations relative to other commonly used methods. For this, we make use of the eight re-simulated galaxies presented in Anglés-Alcázar et al. (2013, 2014) for which two resolution levels are available, corresponding to 20 times and 2.5 times higher mass resolutions relative to our primary simulation in this work. For each of these galaxies, we perform a bulge-disk decomposition at all available redshift snapshots (from early times down to $z=2$ ), according to the following methods.

1. The bulge mass is calculated as double the mass of particles with $v_{\phi}<0$ within the effective radius of the galaxy, where $v_{\phi}$ is the azimuthal velocity of each gas/star particle with respect to the rotation axis of the galaxy (defined as the direction of the total angular momentum within the effective radius). This is the method employed in Anglés-Alcázar et al. (2013) as well as in this work, formally identical to the bulge-disk decomposition procedure used by Abadi et al. (2003).

2. The bulge component corresponds to the total mass of gas/star particles within the effective radius with orbital circularity parameter $\epsilon_{\mathrm{J}} \equiv J_{\mathrm{z}} / J_{\text {circ }}<0.8$, where $J_{z}$ is the gas/star particle component of the specific angular

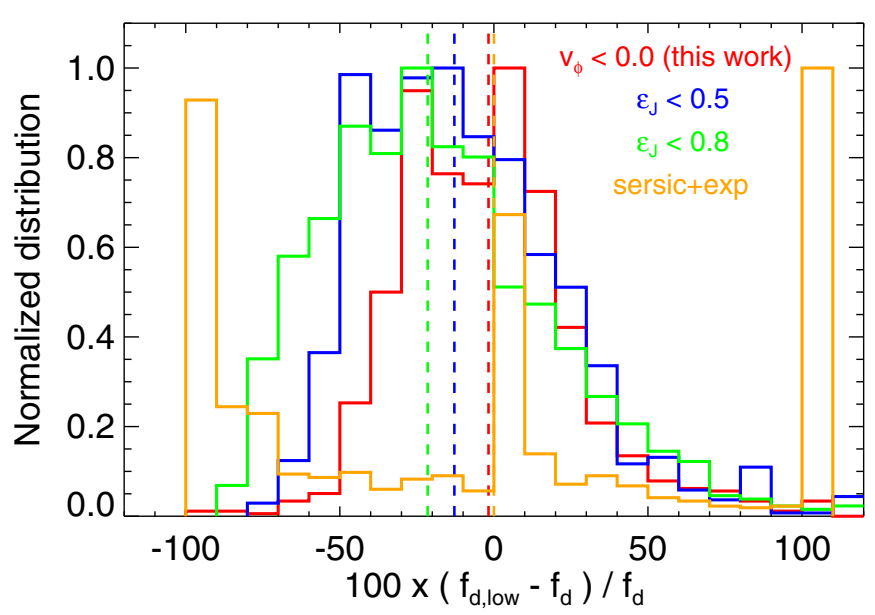

Figure 15. Percentage difference between the disk fractions obtained for the low-resolution $\left(f_{\mathrm{d} \text {,low }}\right)$ and high-resolution $\left(f_{\mathrm{d}}\right)$ zoom-in simulations of AnglésAlcázar et al. $(2013,2014)$ according to different bulge-disk decomposition methods. We calculate $\Delta_{\mathrm{d}}=100 \times\left(f_{\mathrm{d}, \text { low }}-f_{\mathrm{d}}\right) / f_{\mathrm{d}}$ for each galaxy at each time step and plot the normalized distribution of $\Delta_{\mathrm{d}}$ values obtained for all galaxies in the redshift range $z=2-6$. Different criteria for the bulge-disk decomposition are considered, where the bulge mass is calculated as: (1) double the mass of particles with $v_{\phi}<0$ (this work; red), (2) the total mass of particles with $\epsilon_{\mathrm{J}}<0.8$ (green) or $\epsilon_{\mathrm{J}}<0.5$ (blue), and (3) the mass contribution of the Sérsic component from Sérsic+exponential fits to the mass surface density profiles (orange). Vertical dashed lines of different colors indicate median percentage variations for each method. Note that upper and lower limits of $\pm 100 \%$ have been imposed to the results from Sérsic+exponential fitting.

momentum along the rotation axis of the galaxy and $J_{\text {circ }}=$ $r \times \sqrt{G M(<r) / r}$ is the specific angular momentum for a circular orbit at the particle radius $r$ corresponding to the enclosed mass $M(<r)$. This method has been used in, e.g., Governato et al. (2009), Scannapieco et al. (2009) and Christensen et al. (2014). Alternatively, we use a lower cut in the orbital circularity parameter to define the bulge component, $\epsilon_{\mathrm{J}}<0.5$, similar to, e.g., Tissera et al. (2013) and Pedrosa et al. (2014).

3. We perform a standard two-component fit (Sérsic bulge plus exponential disk) to the face-on azimuthally averaged mass surface density profiles of the stellar and gas components of simulated galaxies at all available redshift snapshots. The bulge mass is, then, computed as the integral of the Sérsic profile within the effective radius of the galaxy. Similar profile-fitting decompositions have been extensively applied to idealized galaxy simulations as well as highresolution cosmological simulations (e.g., Robertson et al. 2006a; Hopkins et al. 2009; Christensen et al. 2014).

For all three methods, the disk fraction is calculated as $f_{\mathrm{d}}=$ $1-M_{\text {bulge }}\left(R_{\text {eff }}\right) / M_{\text {tot }}\left(R_{\text {eff }}\right)$, where $M_{\text {bulge }}\left(R_{\text {eff }}\right)$ and $M_{\text {tot }}\left(R_{\text {eff }}\right)$ correspond to the bulge mass and total mass for the gas and stellar components within the effective radius of the galaxy. The resulting disk fractions are, then, compared between the highresolution and low-resolution simulations of each galaxy as a function of redshift. Figure 15 shows the normalized distribution of the percentage difference between the disk fractions obtained for the low- and high-resolution simulations according to the bulge-disk decomposition methods summarized above. As expected given the chaotic nature of hierarchical galaxy formation, we find a significant scatter in the distribution of disk fractions obtained from simulations of different resolution, which, nonetheless, produce galaxy morphologies in overall agreement. We find, however, a mild trend for lower disk fractions in the low-resolution simulations relative to the high-resolution 
simulations, with median percentage variations of $-21 \%$, $-13 \%$, and $-2 \%$ for the $\epsilon_{\mathrm{J}}<0.8, \epsilon_{\mathrm{J}}<0.5$, and $v_{\phi}<0$ bulge-disk decomposition conditions (methods 1 and 2). That is, more strict conditions for the definition of the disk component in galaxies yield increasingly worse resolution convergence, with the simple kinematic decomposition used in this work $\left(v_{\phi}<0\right)$ performing surprisingly well (at the expense of overestimating the disk fraction in the case of rotating bulges). Indeed, our modeling yields good numerical convergence relative to the black hole-galaxy scaling relations, as explicitly shown in AnglésAlcázar et al. (2013). Note that the two-component (Sérsic plus exponential) profile fitting yields very inconsistent results between the disk fractions of high-resolution galaxies and their low-resolution analogs, owing to the degeneracy of fitting parameters, which cannot be appropriately constrained for a limited number of radial bins in low-resolution simulations. While a more in-depth analysis of bulge-disk decomposition methods is beyond the scope of this work, this illustrates the challenge that increasingly complex models pose on the numerical robustness of cosmological simulations. The morphological decomposition procedure adopted in this work has the advantage of great simplicity together with good numerical convergence at the typical resolution of large scale cosmological simulations, making it a very attractive choice for on-the-fly calculations of black hole growth in galaxies across cosmic time.

\section{REFERENCES}

Abadi, M. G., Navarro, J. F., Steinmetz, M., \& Eke, V. R. 2003, ApJ, 597, 21 Abramowicz, M. A., Czerny, B., Lasota, J. P., \& Szuszkiewicz, E. 1988, ApJ, 332,646

Abramowicz, M. A., \& Fragile, C. P. 2013, LRR, 16, 1

Aird, J., Coil, A. L., Moustakas, J., et al. 2012, ApJ, 746, 90

Aird, J., Nandra, K., Laird, E. S., et al. 2010, MNRAS, 401, 2531

Alexander, D. M., \& Hickox, R. C. 2012, NewAR, 56, 93

Anglés-Alcázar, D., Davé, R., Özel, F., \& Oppenheimer, B. D. 2014, ApJ, 782,84

Anglés-Alcázar, D., Özel, F., \& Davé, R. 2013, ApJ, 770, 5

Balbus, S. A., \& Hawley, J. F. 1998, RvMP, 70, 1

Baldry, I. K., Glazebrook, K., \& Driver, S. P. 2008, MNRAS, 388, 945

Barnes, J. E., \& Hernquist, L. 1992, ARA\&A, 30, 705

Begelman, M. C., Volonteri, M., \& Rees, M. J. 2006, MNRAS, 370, 289

Bellovary, J., Volonteri, M., Governato, F., et al. 2011, ApJ, 742, 13

Bellovary, J. M., Governato, F., Quinn, T. R., et al. 2010, ApJL, 721, L148

Bennert, V. N., Auger, M. W., Treu, T., Woo, J.-H., \& Malkan, M. A. 2011, ApJ, 742,107

Blandford, R. D., \& Payne, D. G. 1982, MNRAS, 199, 883

Blecha, L., \& Loeb, A. 2008, MNRAS, 390, 1311

Bogdán, Á., Forman, W. R., Zhuravleva, I., et al. 2012, ApJ, 753, 140

Bondi, H. 1952, MNRAS, 112, 195

Bondi, H., \& Hoyle, F. 1944, MNRAS, 104, 273

Bongiorno, A., Merloni, A., Brusa, M., et al. 2012, MNRAS, 427, 3103

Booth, C. M., \& Schaye, J. 2009, MNRAS, 398, 53

Bournaud, F., Dekel, A., Teyssier, R., et al. 2011, ApJL, 741, L33

Bower, R. G., Benson, A. J., Malbon, R., et al. 2006, MNRAS, 370, 645

Boyle, B. J., \& Terlevich, R. J. 1998, MNRAS, 293, L49

Bu, D.-F., Yuan, F., Wu, M., \& Cuadra, J. 2013, MNRAS, 434, 1692

Cattaneo, A., Faber, S. M., Binney, J., et al. 2009, Natur, 460, 213

Cen, R. 2012, ApJ, 755, 28

Chabrier, G. 2003, PASP, 115, 763

Chen, C.-T. J., Hickox, R. C., Alberts, S., et al. 2013, ApJ, 773, 3

Choi, E., Ostriker, J. P., Naab, T., \& Johansson, P. H. 2012, ApJ, 754, 125

Choi, J.-H., Shlosman, I., \& Begelman, M. C. 2013, ApJ, 774, 149

Christensen, C. R., Brooks, A. M., Fisher, D. B., et al. 2014, MNRAS, 440, L51

Cisternas, M., Jahnke, K., Bongiorno, A., et al. 2011a, ApJL, 741, L11

Cisternas, M., Jahnke, K., Inskip, K. J., et al. 2011b, ApJ, 726, 57

Colberg, J. M., \& Di Matteo, T. 2008, MNRAS, 387, 1163

Comerford, J. M., Gerke, B. F., Newman, J. A., et al. 2009, ApJ, 698, 956

Croton, D. J., Springel, V., White, S. D. M., et al. 2006, MNRAS, 365, 11

Davé, R., Finlator, K., \& Oppenheimer, B. D. 2011a, MNRAS, 416, 1354
Davé, R., Katz, N., Oppenheimer, B. D., Kollmeier, J. A., \& Weinberg, D. H. 2013, MNRAS, 434, 2645

Davé, R., Oppenheimer, B. D., \& Finlator, K. 2011b, MNRAS, 415, 11

Debuhr, J., Quataert, E., \& Ma, C.-P. 2011, MNRAS, 412, 1341

Decarli, R., Falomo, R., Treves, A., et al. 2010, MNRAS, 402, 2453

Dekel, A., Birnboim, Y., Engel, G., et al. 2009, Natur, 457, 451

De Rosa, G., Decarli, R., Walter, F., et al. 2011, ApJ, 739, 56

Di Matteo, T., Colberg, J., Springel, V., Hernquist, L., \& Sijacki, D. 2008, ApJ, 676,33

Di Matteo, T., Springel, V., \& Hernquist, L. 2005, Natur, 433, 604

Dubois, Y., Devriendt, J., Slyz, A., \& Teyssier, R. 2010, MNRAS, 409, 985

Dubois, Y., Devriendt, J., Slyz, A., \& Teyssier, R. 2012, MNRAS, 420, 2662

Dubois, Y., Gavazzi, R., Peirani, S., \& Silk, J. 2013, MNRAS, 433, 3297

Dubois, Y., Volonteri, M., \& Silk, J. 2014, MNRAS, 440, 1590

Elbaz, D., Dickinson, M., Hwang, H. S., et al. 2011, A\&A, 533, 119

Emsellem, E. 2013, MNRAS, 433, 1862

Escala, A. 2006, ApJL, 648, L13

Escala, A. 2007, ApJ, 671, 1264

Fabian, A. C. 2012, ARA\&A, 50, 455

Feltre, A., Hatziminaoglou, E., Hernán-Caballero, A., et al. 2013, MNRAS, 434,2426

Ferrarese, L., \& Merritt, D. 2000, ApJL, 539, L9

Feruglio, C., Maiolino, R., Piconcelli, E., et al. 2010, A\&A, 518, L155

Gabor, J. M., \& Bournaud, F. 2013, MNRAS, 434, 606

Gabor, J. M., \& Davé, R. 2012, MNRAS, 427, 1816

Gabor, J. M., Davé, R., Oppenheimer, B. D., \& Finlator, K. 2011, MNRAS, 417,2676

Gabor, J. M., Impey, C. D., Jahnke, K., et al. 2009, ApJ, 691, 705

Georgakakis, A., Coil, A. L., Laird, E. S., et al. 2009, MNRAS, 397, 623

Gonçalves, T. S., Steidel, C. C., \& Pettini, M. 2008, ApJ, 676, 816

Governato, F., Brook, C. B., Brooks, A. M., et al. 2009, MNRAS, 398, 312

Graham, A. W. 2008, ApJ, 680, 143

Graham, A. W. 2012, ApJ, 746, 113

Graham, A. W., Onken, C. A., Athanassoula, E., \& Combes, F. 2011, MNRAS, 412,2211

Graham, A. W., \& Scott, N. 2013, ApJ, 764, 151

Greene, J. E. 2012, NatCo, 3, 1304

Greene, J. E., Ho, L. C., \& Ulvestad, J. S. 2006, ApJ, 636, 56

Greene, J. E., Peng, C. Y., \& Ludwig, R. R. 2010, ApJ, 709, 937

Gültekin, K., Richstone, D. O., Gebhardt, K., et al. 2009, ApJ, 698, 198

Haardt, F., \& Madau, P. 2001, in Clusters of Galaxies and the High Redshift Universe Observed in X-Rays, ed. D. M. Neumann \& J. T. T. Van (Saclay: CEA Saclay), 64

Häring, N., \& Rix, H. W. 2004, ApJ, 604, 89

Harrison, C. M., Alexander, D. M., Mullaney, J. R., et al. 2012, ApJL, 760, L15

Heckman, T. M., \& Best, P. N. 2014, ARA\&A, 52, 589

Hernquist, L. 1989, Natur, 340, 687

Hickox, R. C., Jones, C., Forman, W. R., et al. 2009, ApJ, 696, 891

Hickox, R. C., Mullaney, J. R., Alexander, D. M., et al. 2014, ApJ, 782, 9

Hinshaw, G., Larson, D., Komatsu, E., et al. 2013, ApJS, 208, 19

Hirschmann, M., Khochfar, S., Burkert, A., et al. 2010, MNRAS, 407, 1016

Hobbs, A., Power, C., Nayakshin, S., \& King, A. R. 2012, MNRAS, 421, 3443

Hopkins, A. M., \& Beacom, J. F. 2006, ApJ, 651, 142

Hopkins, P. F., Bundy, K., Croton, D., et al. 2010, ApJ, 715, 202

Hopkins, P. F., Cox, T. J., Younger, J. D., \& Hernquist, L. 2009, ApJ, 691, 1168

Hopkins, P. F., \& Elvis, M. 2010, MNRAS, 401, 7

Hopkins, P. F., Hernquist, L., Cox, T. J., Robertson, B., \& Krause, E. 2007, ApJ, 669,45

Hopkins, P. F., Hernquist, L., Cox, T. J., et al. 2006, ApJS, 163, 1

Hopkins, P. F., \& Quataert, E. 2010, MNRAS, 407, 1529

Hopkins, P. F., \& Quataert, E. 2011, MNRAS, 415, 1027

Hopkins, P. F., Quataert, E., \& Murray, N. 2012, MNRAS, 421, 3522

Hoyle, F., \& Lyttleton, R. A. 1939, PCPS, 34, 405

Hu, J. 2008, MNRAS, 386, 2242

Jahnke, K., Bongiorno, A., Brusa, M., et al. 2009, ApJL, 706, L215

Jahnke, K., \& Macciò, A. V. 2011, ApJ, 734, 92

Jogee, S. 2006, in AGN Physics on All Scales, ed. D. Alloin, R. Johnson, \& P. Lira (Lecture Notes in Physics, Vol. 93; Berlin: Springer), 143

Juneau, S., Dickinson, M., Bournaud, F., et al. 2013, ApJ, 764, 176

Katz, N., Weinberg, D. H., \& Hernquist, L. 1996, ApJS, 105, 19

Kauffmann, G., \& Heckman, T. M. 2009, MNRAS, 397, 135

Kauffmann, G., Heckman, T. M., Tremonti, C., et al. 2003, MNRAS, 346, 1055

Kelly, B. C., \& Shen, Y. 2013, ApJ, 764, 45

Kennicutt, R. C. 1998, ApJ, 498, 541

Keres, D., Katz, N., Weinberg, D. H., \& Davé, R. 2005, MNRAS, 363, 2

King, A. 2003, ApJL, 596, L27

King, A. L., Miller, J. M., Raymond, J., et al. 2013, ApJ, 762, 103 
Kitayama, T., \& Suto, Y. 1996, ApJ, 469, 480

Kocevski, D. D., Faber, S. M., Mozena, M., et al. 2012, ApJ, 744, 148

Kollmeier, J. A., Onken, C. A., Kochanek, C. S., et al. 2006, ApJ, 648, 128

Kormendy, J., Bender, R., \& Cornell, M. E. 2011, Natur, 469, 374

Kormendy, J., \& Ho, L. C. 2013, ARA\&A, 51, 511

Kulier, A., Ostriker, J. P., Natarajan, P., Lackner, C. N., \& Cen, R. 2013, arXiv: 1307.3684

Lauer, T. R., Tremaine, S., Richstone, D., \& Faber, S. M. 2007, ApJ, 670, 249

Levine, R., Gnedin, N. Y., \& Hamilton, A. J. S. 2010, ApJ, 716, 1386

Li, J., Ostriker, J., \& Sunyaev, R. 2013, ApJ, 767, 105

Lusso, E., Comastri, A., Simmons, B. D., et al. 2012, MNRAS, 425, L623

Lutz, D., Mainieri, V., Rafferty, D., et al. 2010, ApJ, 712, 1287

Maccarone, T. J., Gallo, E., \& Fender, R. 2003, MNRAS, 345, L19

Madau, P., Ferguson, H. C., Dickinson, M. E., et al. 1996, MNRAS, 283, 1388

Madau, P., \& Rees, M. J. 2001, ApJL, 551, L27

Magorrian, J., Tremaine, S., Richstone, D., et al. 1998, AJ, 115, 2285

Maiolino, R., Gallerani, S., Neri, R., et al. 2012, MNRAS, 425, L66

Marconi, A., Risaliti, G., Gilli, R., et al. 2004, MNRAS, 351, 169

Marleau, F. R., Simard, L., Clancy, D., \& Bianconi, M. 2013, MNRAS, 435, 3085

McConnell, N. J., Ma, C.-P., Gebhardt, K., et al. 2011, Natur, 480, 215

McHardy, I. M. 2013, MNRAS, 430, 49L

McNamara, B. R., \& Nulsen, P. E. J. 2007, ARA\&A, 45, 117

Merloni, A., Bongiorno, A., Bolzonella, M., et al. 2010, ApJ, 708, 137

Merloni, A., \& Heinz, S. 2008, MNRAS, 388, 1011

Micic, M., Holley-Bockelmann, K., \& Sigurdsson, S. 2011, MNRAS, 414, 1127

Mullaney, J. R., Daddi, E., Béthermin, M., et al. 2012a, ApJL, 753, L30

Mullaney, J. R., Pannella, M., Daddi, E., et al. 2012b, MNRAS, 419, 95

Murali, C., Katz, N., Hernquist, L., Weinberg, D. H., \& Davé, R. 2002, ApJ, 571,1

Murray, N., Quataert, E., \& Thompson, T. A. 2005, ApJ, 618, 569

Murray, N., Quataert, E., \& Thompson, T. A. 2010, ApJ, 709, 191

Narayanan, D., Cox, T. J., Robertson, B., et al. 2006, ApJL, 642, L107

Narayan, R., \& McClintock, J. E. 2008, NewAR, 51, 733

Narayan, R., \& Yi, I. 1995a, ApJ, 444, 231

Narayan, R., \& Yi, I. 1995b, ApJ, 452, 710

Netzer, H. 2009, MNRAS, 399, 1907

Netzer, H., \& Trakhtenbrot, B. 2007, ApJ, 654, 754

Newton, R. D. A., \& Kay, S. T. 2013, MNRAS, 434, 3606

Novak, G. S., Ostriker, J. P., \& Ciotti, L. 2011, ApJ, 737, 26

Ohsuga, K., \& Mineshige, S. 2011, ApJ, 736, 2

Okamoto, T., Nemmen, R. S., \& Bower, R. G. 2008, MNRAS, 385, 161

Oppenheimer, B. D., \& Davé, R. 2006, MNRAS, 373, 1265

Oppenheimer, B. D., \& Davé, R. 2008, MNRAS, 387, 577

Oppenheimer, B. D., Davé, R., Kereš, D., et al. 2010, MNRAS, 406, 2325

Page, M. J., Symeonidis, M., Vieira, J. D., et al. 2012, Natur, 485, 213

Park, D., Woo, J.-H., Denney, K. D., \& Shin, J. 2013, ApJ, 770, 87

Pedrosa, S. E., Tissera, P. B., \& De Rossi, M. E. 2014, A\&A, 567, 47

Peng, C. Y. 2007, ApJ, 671, 1098

Proga, D., Ostriker, J. P., \& Kurosawa, R. 2008, ApJ, 676, 101

Proga, D., Stone, J. M., \& Kallman, T. R. 2000, ApJ, 543, 686

Puchwein, E., \& Springel, V. 2013, MNRAS, 428, 2966

Rafferty, D. A., Brandt, W. N., Alexander, D. M., et al. 2011, ApJ, 742, 3

Remillard, R. A., \& McClintock, J. E. 2006, ARA\&A, 44, 49

Reynolds, C. S. 1997, MNRAS, 286, 513

Robertson, B., Bullock, J. S., Cox, T. J., et al. 2006a, ApJ, 645, 986

Robertson, B., Hernquist, L., Cox, T. J., et al. 2006b, ApJ, 641, 90

Rodighiero, G., Vaccari, M., Franceschini, A., et al. 2010, A\&A, 515, 8
Rosario, D. J., Santini, P., Lutz, D., et al. 2012, A\&A, 545, A45

Rosario, D. J., Santini, P., Lutz, D., et al. 2013, ApJ, 771, 63

Rovilos, E., Comastri, A., Gilli, R., et al. 2012, A\&A, 546, A58

Rupke, D. S. N., \& Veilleux, S. 2011, ApJL, 729, L27

Sadowski, A., Narayan, R., Penna, R., \& Zhu, Y. 2013, MNRAS, 436, 3856

Santini, P., Rosario, D. J., Shao, L., et al. 2012, A\&A, 540, A109

Scannapieco, C., White, S. D. M., Springel, V., \& Tissera, P. B. 2009, MNRAS, 396, 696

Schawinski, K., Simmons, B. D., Urry, C. M., Treister, E., \& Glikman, E. 2012, MNRAS, 425, L61

Schawinski, K., Thomas, D., Sarzi, M., et al. 2007, MNRAS, 382, 1415

Schmidt, M. 1959, ApJ, 129, 243

Schramm, M., \& Silverman, J. D. 2013, ApJ, 767, 13

Schulze, A., \& Wisotzki, L. 2011, A\&A, 535, A87

Scott, N., Graham, A. W., \& Schombert, J. 2013, ApJ, 768, 76

Shakura, N. I., \& Sunyaev, R. A. 1973, A\&A, 24, 337

Shankar, F., Weinberg, D. H., \& Miralda-Escudé, J. 2013, MNRAS, 428, 421

Shen, Y., \& Kelly, B. C. 2010, ApJ, 713, 41

Shlosman, I., Frank, J., \& Begelman, M. C. 1989, Natur, 338, 45

Silk, J. 2013, ApJ, 772, 112

Silk, J., \& Nusser, A. 2010, ApJ, 725, 556

Silk, J., \& Rees, M. J. 1998, A\&A, 331, L1

Silverman, J. D., Green, P. J., Barkhouse, W. A., et al. 2008, ApJ, 679, 118

Silverman, J. D., Lamareille, F., Maier, C., et al. 2009, ApJ, 696, 396

Simmons, B. D., Lintott, C., Schawinski, K., et al. 2013, MNRAS, 429, 2199

Soltan, A. 1982, MNRAS, 200, 115

Somerville, R. S., Hopkins, P. F., Cox, T. J., Robertson, B. E., \& Hernquist, L. 2008, MNRAS, 391, 481

Springel, V. 2005, MNRAS, 364, 1105

Springel, V., Di Matteo, T., \& Hernquist, L. 2005, ApJL, 620, L79

Springel, V., \& Hernquist, L. 2003, MNRAS, 339, 289

Sturm, E., González-Alfonso, E., Veilleux, S., et al. 2011, ApJL, 733, L16

Sutherland, R. S., \& Dopita, M. A. 1993, ApJS, 88, 253

Targett, T. A., Dunlop, J. S., \& McLure, R. J. 2012, MNRAS, 420, 3621

Teyssier, R., Moore, B., Martizzi, D., Dubois, Y., \& Mayer, L. 2011, MNRAS, 414, 195

Thompson, T. A., Quataert, E., \& Murray, N. 2005, ApJ, 630, 167

Tissera, P. B., Scannapieco, C., Beers, T. C., \& Carollo, D. 2013, MNRAS, 432, 3391

Tombesi, F., Cappi, M., Reeves, J. N., \& Braito, V. 2012, MNRAS, 422, L1

Tombesi, F., Cappi, M., Reeves, J. N., et al. 2013, MNRAS, 430, 1102

Trakhtenbrot, B., \& Netzer, H. 2012, MNRAS, 427, 3081

Trakhtenbrot, B., Netzer, H., Lira, P., \& Shemmer, O. 2011, ApJ, 730, 7

Treister, E., Schawinski, K., Urry, C. M., \& Simmons, B. D. 2012, ApJL, 758, L39

Tremaine, S., Gebhardt, K., Bender, R., et al. 2002, ApJ, 574, 740

Treu, T., Woo, J.-H., Malkan, M. A., \& Blandford, R. D. 2007, ApJ, 667, 117

Trump, J. R., Hsu, A. D., Fang, J. J., et al. 2013, ApJ, 763, 133

Trump, J. R., Impey, C. D., Kelly, B. C., et al. 2011, ApJ, 733, 60

van den Bosch, R. C. E., Gebhardt, K., Gültekin, K., et al. 2012, Natur, 491, 729

Veilleux, S., Cecil, G., \& Bland-Hawthorn, J. 2005, ARA\&A, 43, 769

Volonteri, M. 2010, A\&AR, 18, 279

Volonteri, M., \& Ciotti, L. 2013, ApJ, 768, 29

Wurster, J., \& Thacker, R. J. 2013, MNRAS, 431, 2513

Yu, Q., \& Tremaine, S. 2002, MNRAS, 335, 965

Yuan, F., Bu, D., \& Wu, M. 2012, ApJ, 761, 130

Zubovas, K., Nayakshin, S., King, A., \& Wilkinson, M. 2013, MNRAS, 433, 3079 